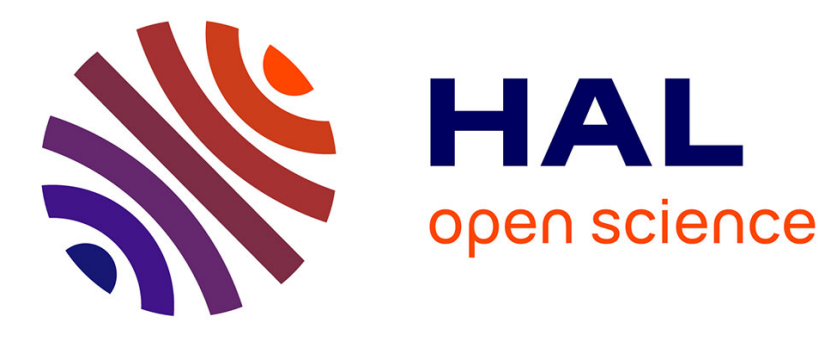

\title{
Active chaotic mixing in a channel with rotating arc-walls
}

Kamal El Omari, Eliane Younes, Teodor Burghelea, Cathy Castelain, Yann Moguen, Yves Le Guer

\section{- To cite this version:}

Kamal El Omari, Eliane Younes, Teodor Burghelea, Cathy Castelain, Yann Moguen, et al.. Active chaotic mixing in a channel with rotating arc-walls. Physical Review Fluids, 2021, 6 (2), 10.1103/PhysRevFluids.6.024502 . hal-03156220

\section{HAL Id: hal-03156220 \\ https://hal.science/hal-03156220}

Submitted on 3 Mar 2021

HAL is a multi-disciplinary open access archive for the deposit and dissemination of scientific research documents, whether they are published or not. The documents may come from teaching and research institutions in France or abroad, or from public or private research centers.
L'archive ouverte pluridisciplinaire $\mathbf{H A L}$, est destinée au dépôt et à la diffusion de documents scientifiques de niveau recherche, publiés ou non, émanant des établissements d'enseignement et de recherche français ou étrangers, des laboratoires publics ou privés. 


\title{
Active chaotic mixing in a channel with rotating arc-walls
}

\author{
Kamal El Omari* \\ Université de Pau et des Pays de l'Adour, E2S UPPA, SIAME, Pau, France and \\ Université de la Réunion, PIMENT, Le Tampon, France \\ Eliane Younes $^{\dagger}$ \\ Université de Pau et des Pays de l'Adour, E2S UPPA, SIAME, Pau, France and \\ LUNAM Université, Université de Nantes, CNRS, \\ Laboratoire de Thermique et Énergie de Nantes UMR 660\%, Nantes, France \\ Teodor Burghelea ${ }^{\ddagger}$ and Cathy Castelain ${ }^{\S}$ \\ LUNAM Université, Université de Nantes, CNRS, \\ Laboratoire de Thermique et Énergie de Nantes UMR 660\%, Nantes, France \\ Yann Moguen ${ }^{\pi}$ and Yves Le Guer** \\ Université de Pau et des Pays de l'Adour, E2S UPPA, SIAME, Pau, France
}

(Dated: March 2, 2021)

\begin{abstract}
An active inline mixer suitable for flows at low Reynolds number and high Péclet number is studied. An alternated oscillatory forcing protocol is imposed by three rotating circular arc-walls in a straight channel. In the two-dimensional case, simple phenomenological arguments are used to estimate heuristically the mixing efficiency with two non-dimensional control parameters: the Strouhal number based on the bulk flow velocity, and the strength of the cross flow relative to the transport flow. The validity and limitations of the proposed mixing conditions are explained by the transport mechanisms in the mixer. The beneficial role of the elliptic flow regions for stretching and folding the passive scalar interfaces is highlighted, as well as a correlation between good mixing ability and the chaotic advection of tracers in the mixing zone.
\end{abstract}

\footnotetext{
*kamal.elomari@univ-pau.fr; kamal.el-omari@univ-reunion.fr

$\dagger$ eliane.younes@univ-nantes.fr

‡ teodor.burghelea@univ-nantes.fr

$\S$ cathy.castelain@univ-nantes.fr

๙ yann.moguen@univ-pau.fr

** yves.leguer@univ-pau.fr
} 


\section{INTRODUCTION}

The efficient transfer of mass or heat in open channel flows of viscous fluids is of importance in many industrial processes: production of food pastes, waste water treatment, pulp and paper manufacturing, inline production of polymer blends with controlled poly-dispersity, etc. In open channel flow mixers, the active zone is continuously fed with the fluids to mix, which spend only a definite time in this zone, in contrast with closed flows mixers. Efficiency is then a crucial issue. To achieve efficient mixing, two categories of open flow mixers can be distinguished, depending on nature of the stirring protocol $[11,31,55]$ : passive or active.

Passive elements use the energy of the flow to generate flow structures enhancing mixing efficiency. This may be carried out by successive changes of direction of the channel walls, for example using twisted pipes [7, 12, 34] or grooved patterns [56]. Passive elements can also move in the flow, such as flexible structures like flaps [2, 38]. Pursuing this idea further, viscoelastic polymers can be added in the fluids to generate a regime of elastic turbulence favorable for mixing [9, 10, 29]. More examples of passive elements are listed in Table I, left column. For a given geometry of an open flow mixer with only passive stirring elements, the bulk velocity is the sole parameter that controls the mixing efficiency. However, for highly viscous fluids, the bulk velocity may be difficult to tune and, consequently, the mixing efficiency is rather limited.

To efficiently mix highly viscous fluids, active elements are expected to perform better. Such elements require an energy source other than the energy associated to the mean flow. Various types of forcing schemes may be used, such as hydrodynamic forcing [8,47] or structural-based forcing (egg-beater [28], rotating walls [45], artificial cilia [18, 36], etc.). More examples are listed in Table I, right column.

\begin{tabular}{l|r}
\hline Passive stirring & Active stirring \\
\hline \hline Converging-diverging channel[3, 25] & Hydrodynamics [8, 47] \\
Rigid or flexible structures[2,38] & Rigid or flexible structures[18, 28, 36, 45] \\
Staggered herringbone channel[56] & Acoustics [14, 33] \\
Partitioned pipe mixer [35] & Electro-hydrodynamics [13] \\
Twisted pipes [7, 12, 34] & Magneto-hydrodynamics [27] \\
Focusing channels [37] & Acoustically driven bubbles [49] \\
Elastic turbulence [9, 10, 29] & Dielectrophoresis [40] \\
Corrugated channel [26] & Electrokinetics [46] \\
\hline
\end{tabular}

TABLE I: Examples of configurations for passive stirring, and types of forcing for active stirring, for open flow mixing (not exhaustive list).

For many active open flow mixers, the mixing is carried out along a channel by moving impeller-like shapes. Yet the mixing length, defined as the characteristic length the fluids need to travel downstream to mix, may be actually smaller than the length of the entire processing line. This can lead to additional energetic costs which would ultimately increase the price of the final product. A second drawback relates to the quality of the final product, like its texture. Many highly viscous pasty materials are made of high molar mass molecular constituents which are mechanically fragile. "Over-mixing" such products may result in mechanical degradation or in a loss of the targeted texture. To circumvent these problems, which have hitherto never been quantitatively addressed [31], certain manufacturers are little inclined to use inline mixers. It is clear that for the latter the ability to easily control the level of mixing is desirable.

Following a preliminary work by two of the authors [20], the central aim of the present paper is to study an active open flow mixer that allows both good mixing efficiency and easy control of the mixing level for highly viscous fluids.

By the large extent of the mixing devices considered above, one can infer that, even in the case of a Newtonian fluid studied in the present paper, the design of the mixer and the choice of the mixing protocol remain challenging tasks. One reason for that is the lack of understanding of the mixing mechanisms, which involve a broad range of spatial and temporal scales and complex interactions between processes at different scales. However, it is generally accepted that in a highly viscous fluid, the mixing of a passive scalar, hereafter referred to as concentration, requires two ordered steps $[19,60]$. The first step is the stretching of the concentration blobs into striations (also called filaments, or lamellae in 2-D) with possibly enhanced diffusion by large shear [24] (also called Taylor dispersion). The second step consists of the folding of the passive scalar filaments allowing for an efficient pure diffusion due to the generated high concentration gradients. One of the simplest mechanisms that permit to achieve the first step is the roll-up of concentration blobs in spirals [43, 52]. Thanks to its rotating arc-walls, the mixer we propose can generate such suitable spirals, provided that the transverse velocity is sufficiently large with respect to the bulk velocity. (What is meant by "sufficiently large" is studied in the present paper.) The step of folding is performed by reorientation of the 
transverse velocity field, namely by the rotational changes of direction of the arc-walls. For simplicity, the rotations of the arc-walls are chosen time periodic. Obviously, an infinite number of protocols could be used to achieve mixing by rotation of the arc-walls. There are practically no theoretical tools available for allowing an a priori choice of a particular form of protocol. Based on qualitative considerations, we thus have chosen a form of protocol that allows us to generate a flow in which hyperbolic and elliptic points coexist, which is recognised as a favorable condition for efficient mixing [53]. We will show that efficient mixing is achieved by a careful choice of the period of rotation and the transverse velocity relative to the bulk velocity. We do not consider here the issue of how to control the mixing level, as done, e.g., by feedback control by Aamo et al. [1], by optimal control by Foures et al. [24], or by entropy maximization by D'Alessandro et al. [15]. The control of mixing often requires a large computational effort. On the contrary, our approach here aims at obtaining choices of control parameters based on extremely simple arguments. For that purpose, a heuristic model based on dimensional and phenomenological arguments will be proposed, giving insights into suitable choices of the control parameters for good mixing.

In the present study a 2-D flow model is considered, as a first step towards understanding of the mixing mechanisms in a realistic 3-D channel mixer. It is recognized that for channel flows, 3-D effects may increase mixing beyond the 2-D mixing mechanisms. Moreover, in 3-D flows, 2-D and 3-D transport mechanisms may be related to each other [48, 51]. This motivates the present 2-D study, which may thus be thought of as giving insights into mixing mechanisms in the channel, despite being only a first step towards understanding of the 3-D mechanisms.

The paper is organised as follows. In section II, the geometry of the proposed mixer is presented, along with the selected flow modulation protocol. In section III, heuristic conditions for mixing, based on dimensional and phenomenological arguments, are proposed. In section IV, the numerical setting-up for two-dimensional simulations is described. Results by the heuristic and numerical approaches are compared in section V. The presence of chaotic advection in the cases of good mixing is evidenced. The paper closes with a summary of the main findings and concluding remarks in section VI.

\section{THE ROTATING ARC-WALL MIXER}

With the objective of mixing efficiently with control of the mixing level, we propose a mixing unit, named Rotating Arc-Wall (or RAW) mixer, that combines the axial flow through a channel and the cross-flow generated by three rotating circular arc-walls.

\section{A. Geometry and kinematics of the RAW mixer}

For the two-dimensional configuration considered for simplicity in the present paper, the RAW mixer consists of a channel of length $L$ and width $W$, with three circular cylinders of equal radii $R$ having their axes of rotation perpendicular to the plane of the channel (see Fig. 1). The cylinders penetrate into the channel with the depth $\Delta$. Two cylinders are located on the same channel wall, at a distance of $2 R$, and one other is located on the opposite wall, in the middle of the two facing cylinders. The three cylinders can rotate independently, with potentially different directions of rotation, with or without temporal modulations of their angular velocities. This flexibility allows a rich variety of flow patterns in the mixer.

\section{B. A protocol for mixing}

As a first step towards the choice of a suitable protocol for the angular velocities of the cylinders, we identify the steady flow topologies that may appear in the RAW mixer without modulation of the angular velocities. Combining the possibilities of rotation of the three cylinders in clockwise and counter-clockwise directions, we obtain $2^{3}$ typical steady-state solutions whose streamlines are shown in Fig. 2. One, two or three elliptic regions [4, 30] appear (see the light-blue zones in Fig. 2), surrounded by separatrices that are transport barriers for the tracers from one elliptic region to another [6]. To break them, the flow is made time-dependent through the rotation of the three cylinders. The modulations of the angular velocities are designed to allow for the reorientation of the transverse velocity field [32]. This is simply achieved by an alternation of the rotation directions. Four couples of steady solutions can then be selected (see Fig. 2) : $\{(1),(2)\},\{(3),(4)\},\{(5),(6)\}$ and $\{(7),(8)\}$, for which the streamlines shown in the figure evolve from one solution to another. At each change of direction, attachment points of the separatrices are either created, removed or destabilized, with a beneficial effect for material transport and thus for mixing. The case of a counter-rotating protocol, in which the cylinders located on the opposite walls rotate in opposite directions, allows the generation of hyperbolic points beneficial to the mixing efficiency. It is also desirable that these points are not 


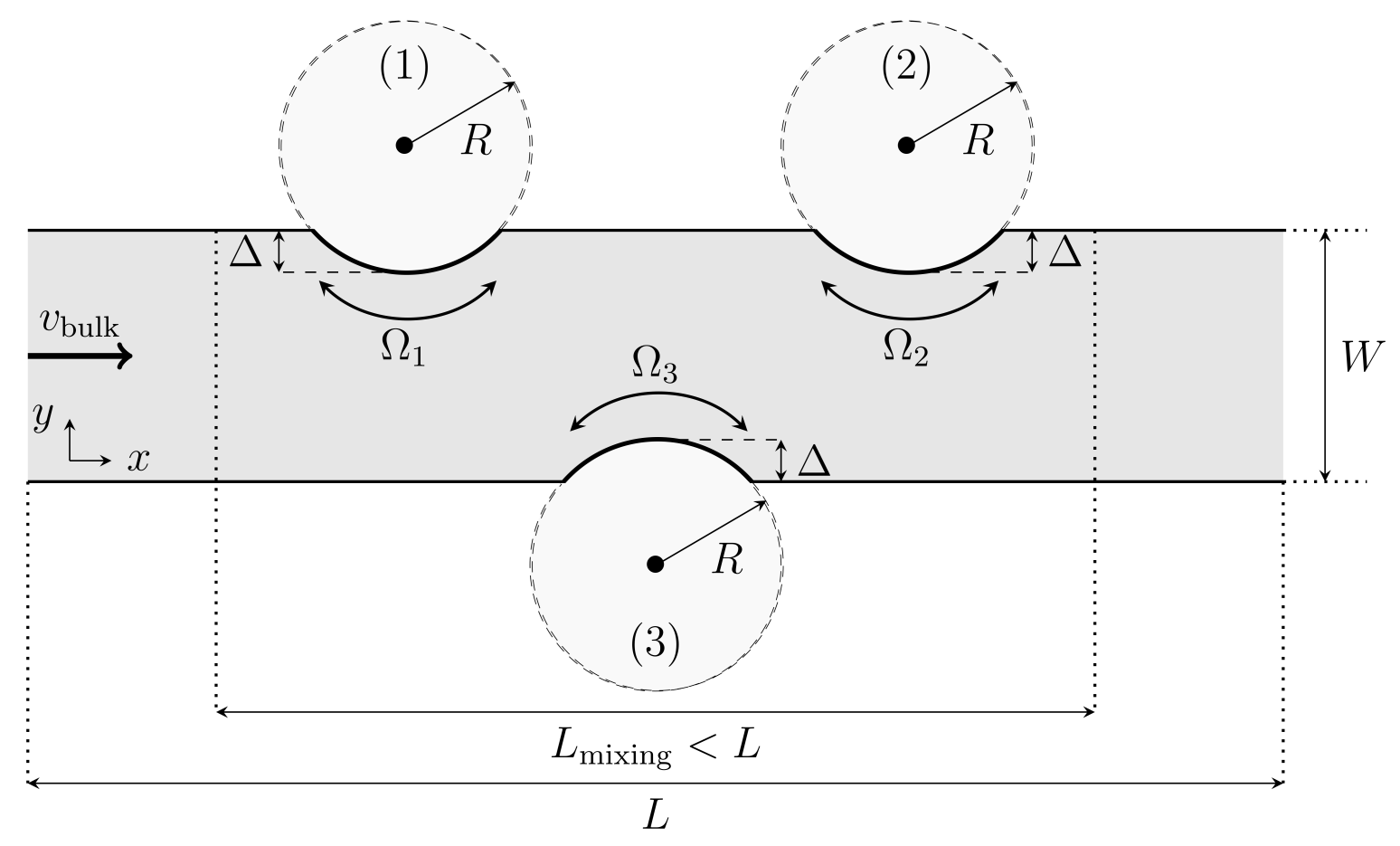

FIG. 1: Sketch of the two-dimensional rotating arc-wall (RAW) mixer (not to scale). $v_{\text {bulk }}$ represents a given bulk velocity of the entering flow.

confined close to the fixed walls [28], but on the contrary are well distributed in the channel, including in the middle area. This leads us to choose equal rotation frequencies and angular velocity amplitudes for the three cylinders. Finally, a smooth alternation between the directions of rotation of the cylinders is chosen so as to reduce the risk of wall slip, with a view to experimental studies of the mixer.

On the basis of many simulations (not shown), a scenario with a smooth alternation of the configurations (7) and (8) in Fig. 2 is selected, and we choose the protocol given by

$$
\Omega_{1}(t)=\Omega_{2}(t)=\Omega_{0} \sin \left(\frac{2 \pi t}{T}\right), \quad \Omega_{3}(t)=-\Omega_{0} \sin \left(\frac{2 \pi t}{T}\right),
$$

where $t$ represents the time, $T$ the forcing period, and $\Omega_{0}$ the amplitude of the angular velocity.

We emphasise that only simple qualitative arguments have been used to end up with Eqs. (1). The form (1) of the protocol allows, depending on the parameter choices, to vary significantly the level of mixing, as will be shown in section $\mathrm{V}$. This will give us the possibility of deducing conclusions of a certain generality, concerning the relationships between flow characteristics and the level of mixing.

\section{HEURISTIC CONDITIONS FOR FOLDING AND MIXING}

In this section, relationships between the dimensions of the RAW mixer and the operating conditions are derived using phenomenological arguments similar to those of Stroock et al. [56] for a passive mixing device, and to those of Ober et al. [45] for an active mixing device. This will provide heuristic conditions for folding and mixing. 
(1)

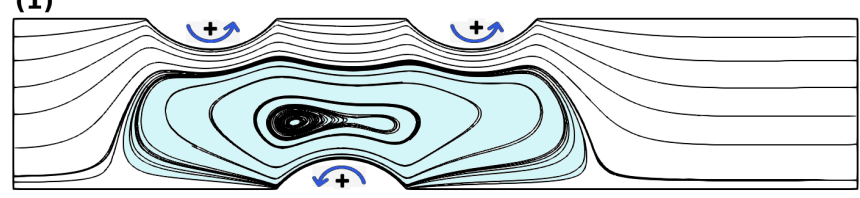

(3)

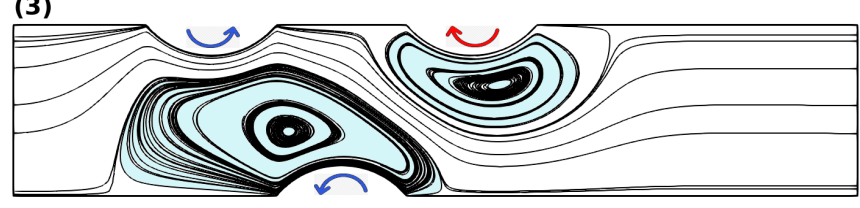

(5)

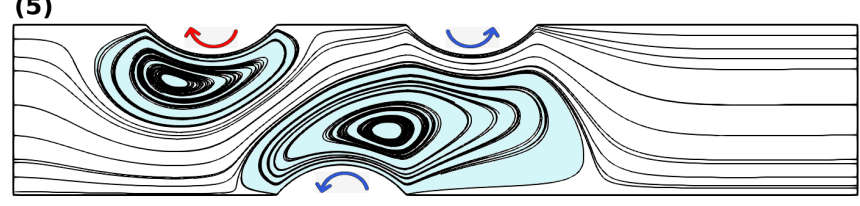

(7)

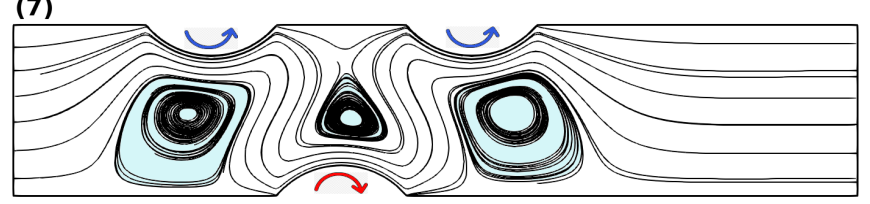

(2)

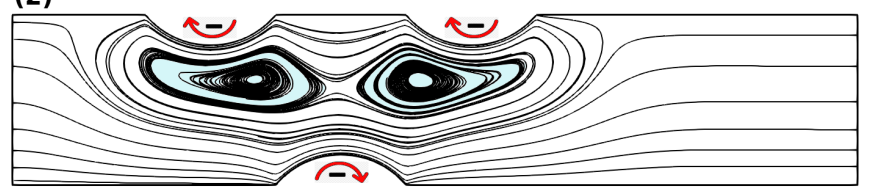

(4)

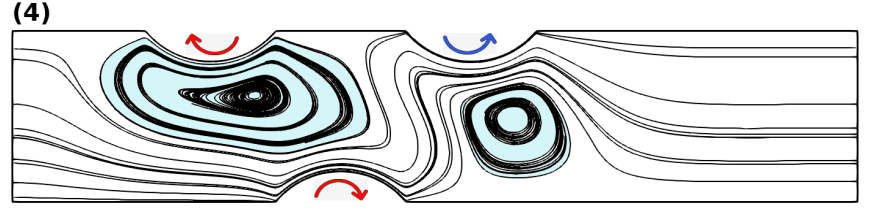

(6)

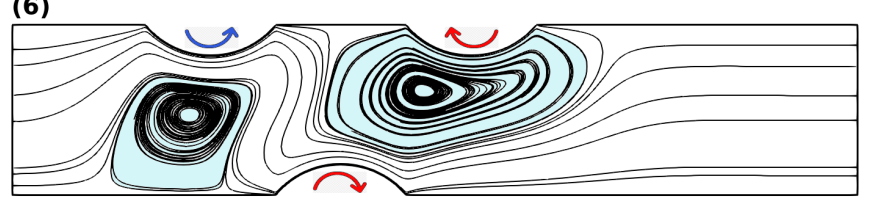

(8)

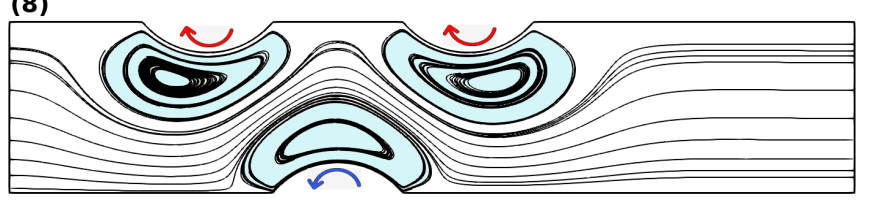

FIG. 2: Typical steady-state streamline flow topologies for the three cylinders rotating at constant angular velocity.

The eight possible stirring configurations are $1(+++), 2(---), 3(++-), 4(--+), 5(-++), 6(+--)$,

$7(+-+)$, and $8(-+-)$. The symbol $(+)$ indicates counter-clockwise direction of the angular velocity of the rotating cylinder (blue arrows), and (-) clockwise direction (red arrows). The elliptic zones are colored in light blue. Here we took $\operatorname{Re}_{\text {bulk }}=1$ and $K_{0}=10$; see section III A.

\section{A. Non-dimensional numbers}

With the given bulk velocity $v_{\text {bulk }}$ as reference velocity and the width channel $W$ as reference length (see Fig. 1 ), a bulk Reynolds number is defined as

$$
\operatorname{Re}_{\mathrm{bulk}}=\frac{v_{\mathrm{bulk}} W}{\nu},
$$

where $\nu$ is the fluid viscosity, and a bulk Péclet number is defined as

$$
\mathrm{Pe}_{\mathrm{bulk}}=\frac{v_{\mathrm{bulk}} W}{\mathcal{D}},
$$

where $\mathcal{D}$ is the molecular diffusivity. Next, introducing a characteristic cross-flow velocity denoted by $v_{\text {cross }}$, a Reynolds number associated to the channel cross flows is defined as

$$
\operatorname{Re}_{\text {cross }}=\frac{v_{\text {cross }} W}{\nu} .
$$

The ratio

$$
K=\frac{\operatorname{Re}_{\text {cross }}}{\operatorname{Re}_{\text {bulk }}}=\frac{v_{\text {cross }}}{v_{\text {bulk }}},
$$


which represents the strength of the cross-flow velocity to the bulk velocity, will be used below, along with the bulk Strouhal number defined as

$$
\mathrm{St}_{\text {bulk }}=\frac{W}{T v_{\text {bulk }}} .
$$

Our choice for the definition of $v_{\text {cross }}$ is given later.

\section{B. Condition for folding}

The stirring mechanism begins when the interface between the two different values of the concentration, assumed to be initially located at mid-width of the channel, is deformed toward the walls by the action of the cylinders rotation. Then, mixing efficiency depends on the relative strength of the bulk and cross-flow velocities. If the cross-flow velocity is too low with respect to the bulk velocity, the deformation of the interface will be almost suppressed by the next rotational change of direction. In such a case, no folding operation is realised. Otherwise, the pulled thread is long enough to be folded by the next rotation of the cylinders in the opposite direction. In terms of displacement, a minimal condition for achieving a folding of the interface is that the characteristic transverse displacement during one half period, $v_{\text {cross }} T / 2$, of a tracer initially located near a rotating arc-wall, exceeds the half-channel width $W / 2$. In this case, however, the length of the generated striations can be negligibly small. To avoid this situation, we rather consider the condition of a characteristic displacement during a half period larger than the full channel width $W$. The length of the generated striations is then larger than the half-channel width. This is a reasonable condition to allow successive foldings of the concentration interface. With Eq. (6), this results in

$$
K \geq 2 \mathrm{St}_{\text {bulk }} .
$$

To obtain from (7) a manageable condition, we have to clarify our choice for the value of $v_{\text {cross }}$. Since the condition (7) concerns the transverse displacement of a tracer during one half period $T / 2$, a definition of the characteristic cross-flow velocity $v_{\text {cross }}$ that is relevant here is by averaging the angular velocity on this duration. Eq. (5) and the condition (7) then give

$$
K_{0} \geq \pi \mathrm{St}_{\text {bulk }},
$$

where $K_{0}$ is defined by

$$
K_{0}=\frac{R \Omega_{0}}{v_{\text {bulk }}} .
$$

The inequality (8) provides guidance on a minimal condition for folding needed to achieve mixing. However, as will be illustrated in section V A, this is only a necessary (but not sufficient) condition. Another condition that takes into account the diffusion process, thus complementary to the condition (8), is discussed in the next section.

\section{Condition for mixing}

If the cross-flow velocity is large enough with respect to the bulk velocity, then the successive changes of direction of the rotating cylinders can generate stretched and folded lamellae of the concentration values down to spatial scales

$$
d=\frac{W}{2^{n}} .
$$

Since the interface between the concentration values is initially located at mid-width of the channel, the number of cycles of stretching and folding operations is $n-1$, with $n \geq 1$. The characteristic time needed for the diffusion to be effective after $n-1$ cycles is then

$$
t_{\text {diffusion }}=\frac{d^{2}}{\mathcal{D}}=\frac{W^{2}}{4^{n} \mathcal{D}} .
$$


A phenomenological condition for the diffusion to be effective after $n-1$ cycles is

$$
t_{\text {residence }} \geq t_{\text {diffusion }}
$$

with the characteristic residence time defined as

$$
t_{\text {residence }}=\frac{L_{\text {mixing }}}{v_{\text {bulk }}}
$$

where $L_{\text {mixing }}$ is the length of the active mixing zone (see Fig. 1). Using the bulk Péclet number of Eq. (3), the characteristic residence time can be written as

$$
t_{\text {residence }}=\frac{W L_{\text {mixing }}}{\mathcal{D} \text { Pe } e_{\text {bulk }}} .
$$

With the diffusion time given by Eq. (11) and the residence time given by Eq. (14), the mixing condition (12) then becomes

$$
n \geq \frac{1}{\ln (4)} \ln \left(\frac{\mathrm{Pe}_{\mathrm{bulk}}}{\zeta}\right)
$$

where $\zeta=L_{\text {mixing }} / W$ is the non-dimensional extent of the mixing zone. Noting that under the folding condition of section III B,

$$
\zeta K=\frac{t_{\text {residence }} v_{\text {cross }}}{W} \geq n
$$

the condition (15) gives

$$
K \geq \frac{1}{\zeta \ln (4)} \ln \left(\frac{\mathrm{Pe}_{\mathrm{bulk}}}{\zeta}\right)
$$

Since in (16) $t_{\text {residence }} v_{\text {cross }}$ is the transverse displacement of a tracer during the characteristic residence time $t_{\text {residence }}$, a relevant definition of the characteristic cross-flow velocity $v_{\text {cross }}$ in (17) is now by averaging the angular velocity on $t_{\text {residence: }}$

$$
v_{\text {cross }}=R \bar{\Omega}, \quad \text { with } \quad \bar{\Omega}=\frac{1}{t_{\text {residence }}} \int_{0}^{t_{\text {residence }}} \Omega_{0}\left|\sin \left(\frac{2 \pi t}{T}\right)\right| \mathrm{d} t .
$$

We shall find it useful to write the characteristic residence time as

$$
t_{\text {residence }}=N T+\delta t
$$

where $0 \leq \delta t<T$. Then,

$$
\bar{\Omega}= \begin{cases}\frac{\Omega_{0}}{\pi \zeta \mathrm{St}_{\text {bulk }}}\left[2 N+\sin ^{2}\left(\pi \zeta \mathrm{St}_{\text {bulk }}\right)\right] & , \text { if } 0 \leq \delta t \leq \frac{T}{2}, \\ \frac{\Omega_{0}}{\pi \zeta \mathrm{St}_{\text {bulk }}}\left[2(N+1)-\sin ^{2}\left(\pi \zeta \mathrm{St}_{\text {bulk }}\right)\right] & , \text { if } \frac{T}{2}<\delta t<T .\end{cases}
$$

Noting that $2(N+1)-\sin ^{2}\left(\pi \zeta \mathrm{St}_{\text {bulk }}\right) \geq 2 N+\sin ^{2}\left(\pi \zeta \mathrm{St}_{\text {bulk }}\right)$, the inequality

$$
\bar{\Omega} \geq \frac{\mathcal{D}}{R W \ln (4)} \frac{\mathrm{Pe}_{\text {bulk }}}{\zeta} \ln \left(\frac{\text { Pe }}{\zeta}\right)
$$

which is equivalent to (17), is satisfied by both expressions in (20) when the following mixing condition is fulfilled:

$$
K_{0} \geq \frac{\pi \mathrm{St}_{\mathrm{bulk}}}{\ln (4)} \frac{\ln \left(\frac{\mathrm{P}_{\mathrm{bulk}}}{\zeta}\right)}{2 \zeta \mathrm{St}_{\mathrm{bulk}}+\sin ^{2}\left(\pi \zeta \mathrm{St}_{\mathrm{bulk}}\right)} .
$$




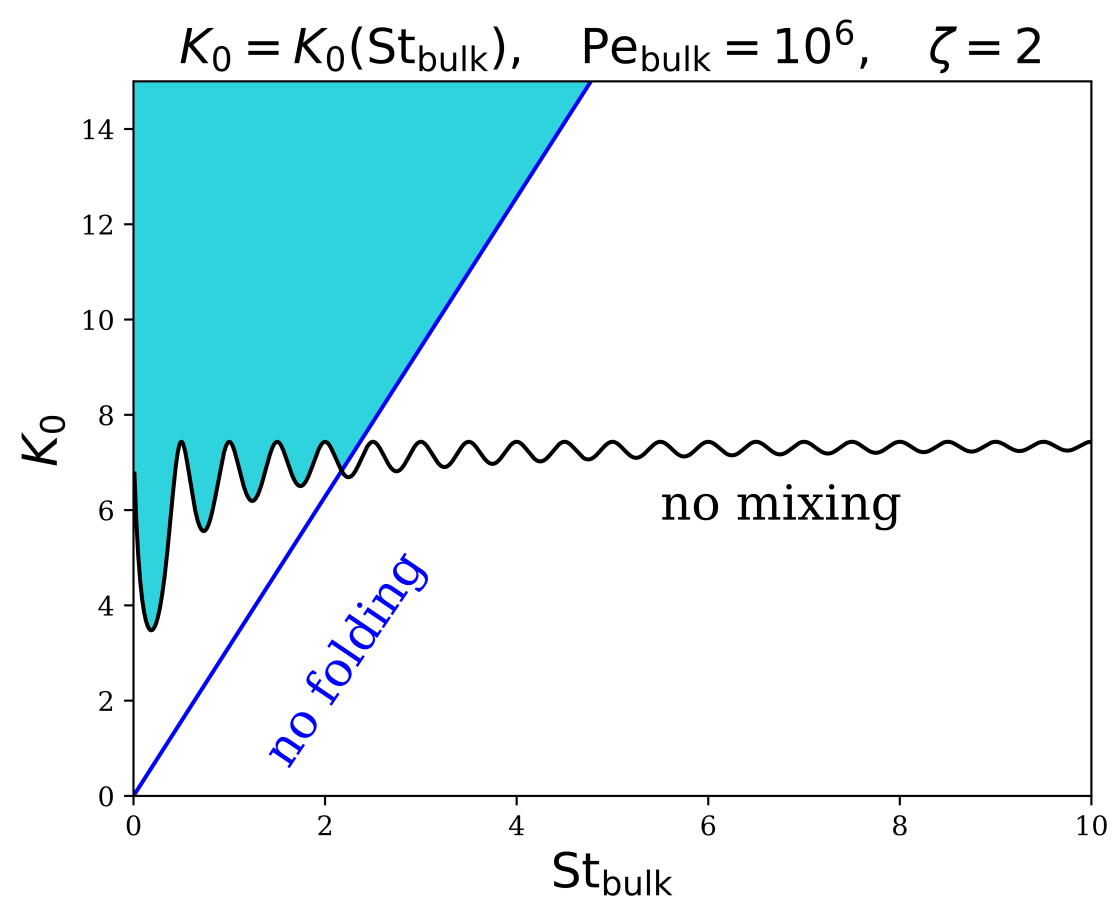

FIG. 3: Region of possible mixing (colored) in the parameter space $\left(\mathrm{St}_{\mathrm{bulk}}, K_{0}\right)$, based on the folding condition (8) (blue line) and the mixing condition (22) (black line). Here, we take $\zeta=2$ and Pe $_{\text {bulk }}=10^{6}$. The region below the black line corresponds to values for which mixing is not expected. The region below the blue line corresponds to values for which folding is not expected. Note that the black line, which represents the mixing condition, has the vertical asymptote $\mathrm{St}_{\mathrm{bulk}}=0$.

The inequality (22) may be thought of as a guidance on a necessary condition for mixing, at any given value of the bulk Péclet number Pe bulk. An example of obtained separating curve in the parameter plane $\left(\mathrm{St}_{\mathrm{bulk}}, K_{0}\right)$ is shown in Fig. 3 (black line). Below the curve are values of $\mathrm{St}_{\text {bulk }}$ and $K_{0}$ that can be considered unfavorable for mixing. Accounting for both conditions (8) and (22), values of $\mathrm{St}_{\text {bulk }}$ and $K_{0}$ that would allow mixing may be determined (coloured zone in Fig. 3). However, at this point we cannot strictly guarantee mixing for such values. This issue is investigated through numerical experiments in the next sections, by comparing the results obtained from the heuristic conditions (8) and (22) to those obtained by direct simulations of the mixing process.

\section{NUMERICAL SETUP}

In this section are presented the settings and the numerical method used for the direct simulations to be compared to the heuristic conditions (8) and (22) for folding and mixing.

\section{A. Equations for the velocity and concentration fields}

For the system we are interested in, the RAW mixer with a Newtonian fluid, we choose the channel width $W$ as length scale, and the forcing period $T$ as time scale. The amplitude of tangential velocity near the rotating arcs $v_{0}=R \Omega_{0}$ is chosen as velocity scale. A viscous scaling is taken for the pressure, with $\mu v_{0} / W$. Assuming an incompressible flow, we thus need to solve, here in a two-dimensional domain, the continuity equation

$$
\nabla \cdot \boldsymbol{v}=0
$$

the momentum equation

$$
\operatorname{Re}_{0}\left[\mathrm{St}_{0} \partial_{t} \boldsymbol{v}+(\boldsymbol{v} \cdot \nabla) \boldsymbol{v}\right]=-\nabla p+\nabla^{2} \boldsymbol{v}
$$


and the equation governing the advection and diffusion of the concentration $c$,

$$
\mathrm{St}_{0} \partial_{t} c+\boldsymbol{v} \cdot \nabla c=\frac{1}{\mathrm{Pe}_{0}} \Delta c .
$$

In the dimensionless equations (23)-(25), $\boldsymbol{v}$ is the velocity, $p$ is the pressure, and

$$
\operatorname{Re}_{0}=\frac{v_{0} W}{\nu}, \quad \mathrm{St}_{0}=\frac{W}{T v_{0}}, \text { and } \mathrm{Pe}_{0}=\frac{v_{0} W}{\mathcal{D}} .
$$

We conclude that for a fixed value of the Péclet number $\mathrm{Pe}_{0}$, two independent non-dimensional parameters in Eqs. (23)-(25) are sufficient for describing the system: $\operatorname{Re}_{0}$ and $\mathrm{Re}_{0} \mathrm{St}_{0}$.

Turning to the inlet conditions, two fluid streams with two distinct concentration levels, $c=0$ and $c=1$, are evenly injected into the channel. The velocity field at the inlet is taken in the form of a Poiseuille profile with $\operatorname{Re}_{\text {bulk }}=v_{\text {bulk }} W / \nu=1$. Thus $\operatorname{Re}_{0}=\operatorname{Re}_{0} / \operatorname{Re}_{\text {bulk }}=K_{0}$ and $\operatorname{Re}_{0} \mathrm{St}_{0}=W^{2} /(\nu T)=\operatorname{Re}_{\text {bulk }} \mathrm{St}_{\text {bulk }}=\mathrm{St}_{\text {bulk }}$. From the above non-dimensionalization, we conclude that $K_{0}$ and $\mathrm{St}_{\text {bulk }}$ are two independent parameters able to describe the system. Their relevance as control parameters of the RAW mixer in the folding and mixing conditions obtained in sections III B and III C is thus confirmed.

At the initial time $t=0$, the interface between the two fluid streams is placed at the middle of the channel, along the $x$-axis (see Fig. 4a). As boundary conditions we use no slip conditions at both the static and moving walls.

\section{B. Dimensions, numerical method, and mixing indicator}

With the channel width $W$ as reference length scale, the non-dimensional geometrical parameters of the domain are as follows. The channel length is 5, the radius of the cylinders is 0.5 , and their penetration depth is 0.167.

The used numerical method is based on a finite-volume formulation with co-located variable arrangement on unstructured meshes, implemented in the in-house code TAMARIs. For technical details, the interested reader is referred to $[5,21,22,39]$. Pressure and velocity are coupled using the SIMPLE algorithm combined with a momentum interpolation method. The transient terms in Eqs. (24) and (25) are discretised using the second-order backward Euler scheme (BDF2). The diffusion, convection and pressure terms are discretised using second-order accurate schemes. For the discretisation of the convection fluxes, the nonlinear CUBISTA scheme is chosen for its low numerical diffusion allowing for accurate representation of the concentration transport.

Generally speaking, in fluid flow simulations the effective overall diffusion encompasses the molecular diffusion in the fluid, which is often prescribed as a numerical setting, and the numerical diffusion inherent to the chosen schemes, mesh size and CFL number, which is often difficult to assess. In the results shown hereafter, the molecular diffusion

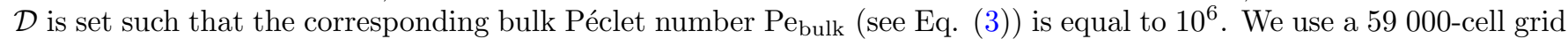
with $\mathrm{CFL}=0.7$. The effective Péclet number, which is thus less than $10^{6}$ due to the numerical diffusion, is the same in all simulations. Thus, we do not focus on understanding the dependence of the mixing efficiency on the effective Péclet number.

The measure of the level of homogeneity of a mixture at finite Péclet number often requires complementary approaches and depends on the relative importance of advection and diffusion processes [16, 23, 24, 41, 42, 59]. In the present work, we adopt for its simplicity and relevance at finite Péclet number the widely accepted measure by the standard deviation of the concentration $c$,

$$
\sigma=\sqrt{\frac{1}{\sum_{I} a_{I}} \sum_{I} a_{I}\left(c_{I}-c_{\text {mean }}\right)^{2}},
$$

with $c_{I}$ the concentration in cell $I, a_{I}$ the area of cell $I$, and $c_{\text {mean }}=\sum a_{I} c_{I} /\left(\sum_{I} a_{I}\right)$. The standard deviation $\sigma$ is evaluated on a rectangular area of non-dimensional width 0.285 distributed over the entire width of the canal downstream of the three cylinders; its location is shown in Fig. 4a.

\section{RESULTS}

In this section, the basic mixing mechanisms in the proposed RAW mixer are first described. Then, the mixing efficiency obtained by the phenomenological and numerical approaches are compared. 


\section{A. Typical patterns of the concentration}

A typical evolution of the two-dimensional field of the concentration in the RAW mixer is shown in Fig. 4, where $\mathrm{St}_{\text {bulk }}=1$ and $K_{0}=20$. If we refer to the parameter plane in Fig. 3, these values of the parameters are clearly favorable for mixing from the heuristic conditions (8) and (22). Indeed, as can be observed in Fig. 4, the cross-flow velocity is sufficiently large with respect to the bulk velocity for complex crossing trajectories to be generated, along with backward flows. Thus, in this case, the heuristic conditions and the direct simulation are in agreement in terms of mixing efficiency.

As shown in Fig. 5, the concentration field is approximately periodic after about 10 periods of rotation of the cylinders. Then, the standard deviation of the concentration oscillates around a constant value (not shown). Recurrent patterns clearly appear after few periods of rotation of the cylinders (compare Figs. 4e and 4f), as observed by Gouillart et al. [28] for another open flow active mixer. Few non mixed regions do remain at the exit of the mixer, as shown in Figs. 4 (see the right boundary of each frame) and 5b. Note that the RAW mixer with three cylinders shown in Fig. 1 can be considered as a single stirring cell unit. Thus, if the level of mixing is not satisfactory, it can be increased by lining up other RAW stirring units.

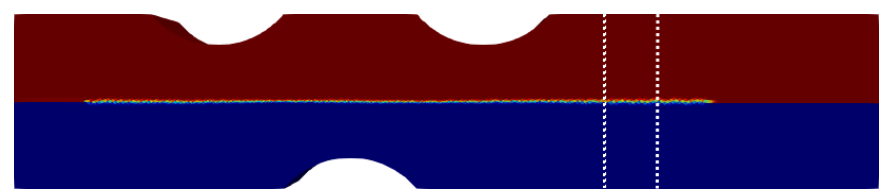

(a) $t=0$.

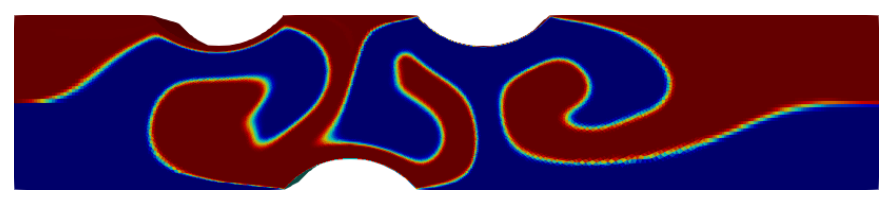

(c) $t=T / 2$.

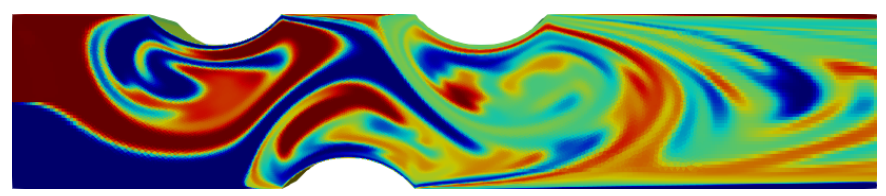

(e) $t=10 T$

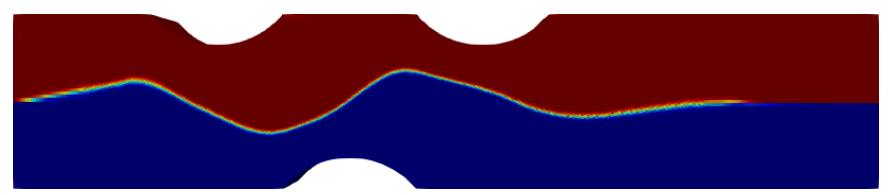

(b) $t=T / 8$.

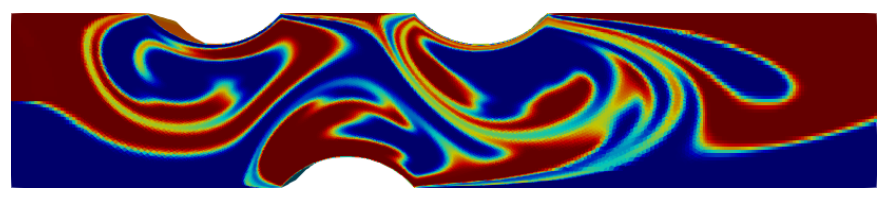

(d) $t=T$.

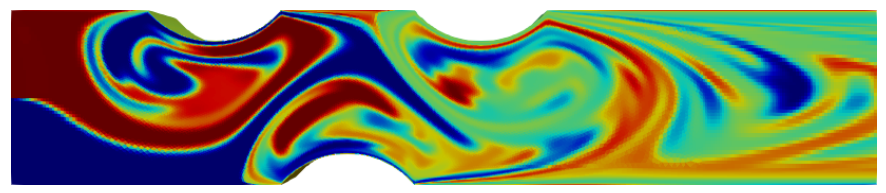

(f) $t=50 T$

FIG. 4: Evolution of the concentration $c$ along the RAW mixer, for $\mathrm{St}_{\text {bulk }}=1$ and $K_{0}=20$. Based on the bulk velocity $v_{\text {bulk }}$, the duration needed for a tracer to cross the mixer is $5 T$. The solution may be considered as approximately periodic after about 10 periods of rotation of the cylinders (see Figs. 4e and 4f). The colour code is: blue for $c=0$, yellow for $c=0.5$ and red for $c=1$. In Fig. 4a, the white dashed lines indicate the rectangular area where the standard deviation $\sigma$ of the concentration is evaluated.

We now describe the basic mixing mechanisms in the RAW mixer. Considering for clarity the first period of cylinder rotation, this period can be divided into two phases (see Figs. 4c and 4d, to be compared to Figs. 2(7) and 2(8), respectively). During the first phase, which corresponds to the first half period, three uniform concentration regions are positioned opposite the rotating cylinders on the three elliptic regions visible in Fig. 2(7). The corresponding three uniform concentration regions take the form of anvils (red, blue, then red again), separated by narrower areas each consisting of two bands of different concentrations (see Fig. 4c). In subsequent periods, concentration regions are no longer monochrome, but made up of increasingly complex concentration gaps. However, the mechanism remains basically the same: During each first half period, the three elliptic regions serve as "waiting rooms" for concentration areas, before stretching them during the second half period. But before this second phase, the cylinders change direction of rotation at the half period. At that time, the elliptic regions present during the first half period disappear and the concentration areas that were on the elliptic regions are advected by the longitudinal flow. Then, as the angular velocity of the cylinders increases, three new elliptic regions emerge near the cylinders (see Fig. 2(8)); the concentration areas that were in the three elliptic regions during the first half period are now entrained between the rotating cylinders and the new elliptic regions. As these regions are very close to the cylinders, the concentration areas are then strongly stretched. In addition, the bands of concentrations separating the areas above mentioned are wound inside the new elliptic regions. Striations of different concentration levels are then generated. We thus observe 
that the combined action of elliptic regions close to the walls is a key condition for mixing in the RAW mixer, since they allow for the generation of concentration filaments and their folding. The RAW mixer then takes advantage of the possible beneficial role of elliptic regions for mixing in open flows [17].

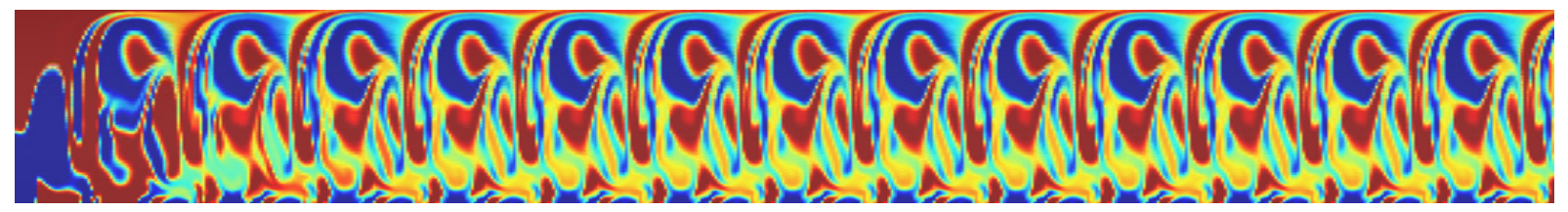

(a)

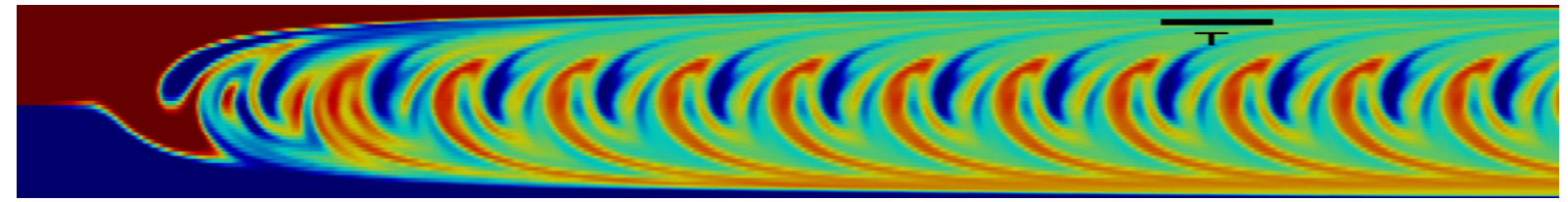

(b)

FIG. 5: Spatio-temporal plots of the concentration $c$ as a function of the time $t$ (abscissa) and the channel height $y$ (ordinate), for $\mathrm{St}_{\mathrm{bulk}}=1$ and $K_{0}=20$. The colour code is: blue for $c=0$, yellow for $c=0.5$ and red for $c=1$. Two cross-sections located at different axial coordinates $x$ are considered along the channel of the RAW mixer: (a) cross-section passing by the lower cylinder axis (see Fig. 1); (b) outlet cross-section. The concentration data are recorded from time $t=0$ to time $t=13.75 T$ for both frames. The black segment in Fig. 5b represents the duration of one period $T$.

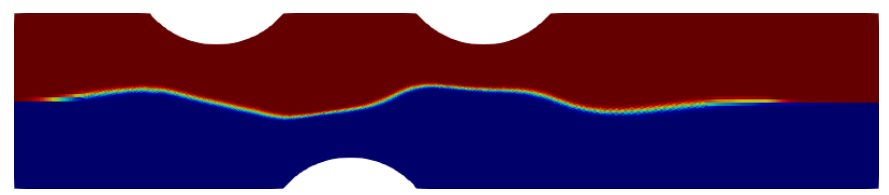

(a) $t=T / 2$

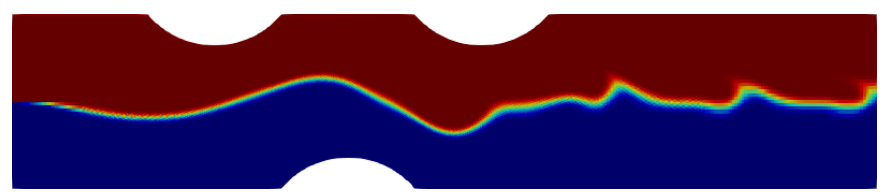

(c) $t=10 T$

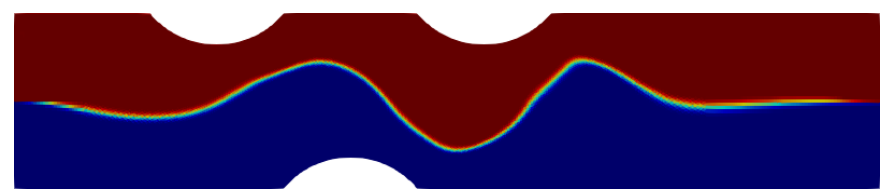

(b) $t=T$.

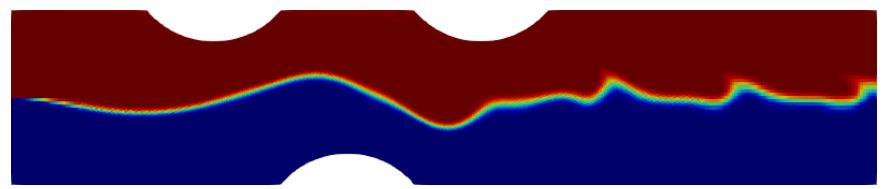

(d) $t=50 T$.

FIG. 6: Evolution of the concentration along the RAW mixer for $\mathrm{St}_{\mathrm{bulk}}=2$ and $K_{0}=5$. This case is unfavorable for mixing if we refer to the parameter plane in Fig. 3. Based on the bulk velocity $v_{\text {bulk }}$, the duration needed for a tracer to cross the mixer is $10 T$. The color code is: blue for $c=0$, yellow for $c=0.5$ and red for $c=1$.

We now consider the parameters $\mathrm{St}_{\text {bulk }}=2$ and $K_{0}=5$, which are, referring to Fig. 3, unfavorable for mixing. Indeed, no mixing is observed in Fig. 6, except in a thin area in the vicinity of the interface between the values 0 and 1 of the concentration. The concentration patterns in Figs. 4 and 6 are completely different. First, the shapes of the interfaces are almost identical in Figs. 4d, 4e and $4 \mathrm{f}$ (despite the fact that the values of the concentration fields in Fig. $4 \mathrm{~d}$ are different from the values in Figs. 4e or 4f). However, the shape of the interface in Fig. 6b is different from the shapes in Figs. 6c or 6d. This means that, in such a case unfavorable for mixing, the duration needed to observe an approximately periodic regime is longer than in case favorable for mixing. Second, in the case favorable for mixing (Fig. 4), the interface is pushed to the walls thanks to the transversal velocity component which is sufficiently large with respect to the bulk velocity in that case. There is a noticeable difference from the results shown in Fig. 6, where the interface remains confined near the middle of the channel.

According to the parameter plane in Fig. 3, a parameter choice less favorable for mixing than $\left(\mathrm{St}_{\text {bulk }}=1, K_{0}=20\right)$ but more favorable than $\left(\mathrm{St}_{\text {bulk }}=2, K_{0}=5\right)$ is $\left(\mathrm{St}_{\text {bulk }}=2, K_{0}=20\right)$. It is apparent in Fig. 7 that the characteristic displacement of a tracer located near the rotating cylinders (2) and (3) during a half period is larger than the half channel width. Note that the requirement of this minimal displacement was used in section IIIC for the derivation 


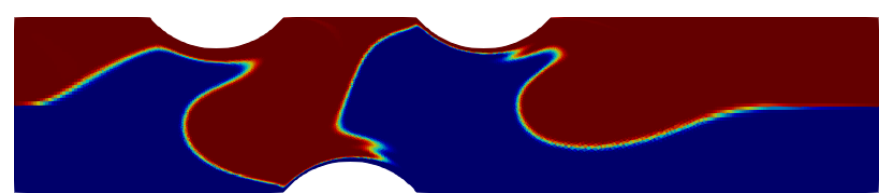

(a) $t=T / 2$.

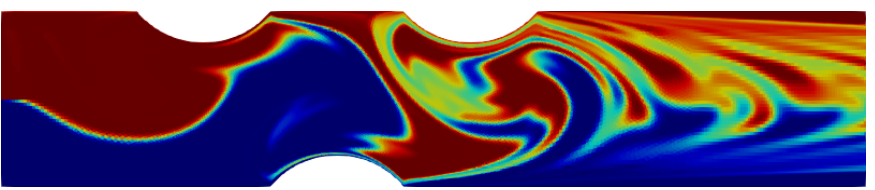

(c) $t=10 T$.

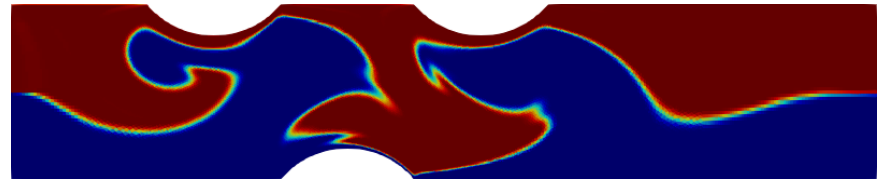

(b) $t=T$.

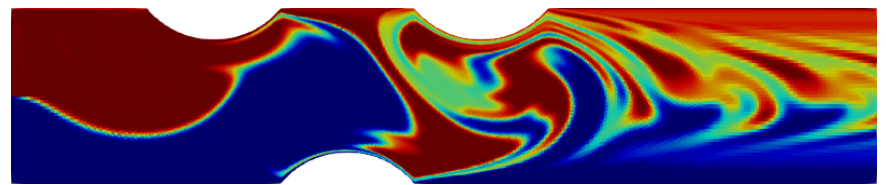

(d) $t=50 T$.

FIG. 7: Evolution of the concentration along the RAW mixer for $\mathrm{St}_{\mathrm{bulk}}=2$ and $K_{0}=20$. This case is in between favorable and unfavorable for mixing if we refer to the parameter planes in Figs. 3 and 13. Based on the bulk velocity $v_{\text {bulk }}$, the duration needed for a tracer to cross the mixer is $10 T$. The color code is: blue for $c=0$, yellow for $c=0.5$ and red for $c=1$.

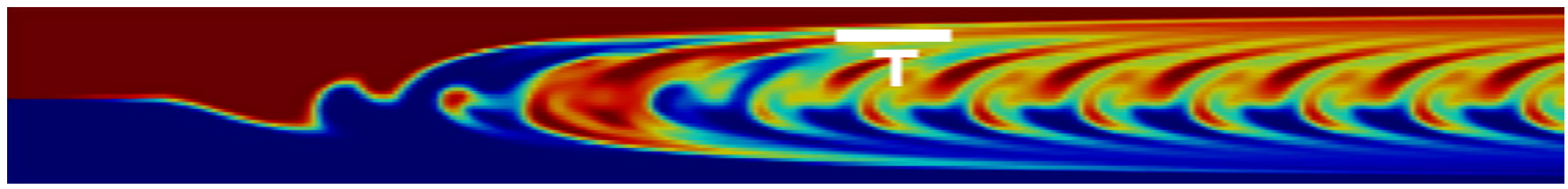

FIG. 8: Spatio-temporal plot of the concentration $c$ as a function of the time $t$ (abscissa) and the channel height $y$ (ordinate), for $\mathrm{St}_{\text {bulk }}=2$ and $K_{0}=20$, at the outlet cross-section of the channel. The concentration data are recorded from time $t=0$ to time $t=13.75 \mathrm{~T}$. The white segment represents the duration of one period $T$. The color code is: blue for $c=0$, yellow for $c=0.5$ and red for $c=1$.

of the mixing condition (22). However, it is clear from Figs. 7d and 8 that this condition is insufficient for mixing, since the mixing level is relatively low at the outlet of the channel. Although large transverse displacements of the tracers are observed, the deformations of the interface are somewhat canceled out by the sequence of rotations of the cylinders.

Finally, since the black line in Fig. 3, which represents the mixing condition (22), has the vertical asymptote $\mathrm{St}_{\text {bulk }}=0$, it is of interest to consider an example with $\mathrm{St}_{\text {bulk }} \ll 1$. We thus take $\mathrm{St}_{\text {bulk }}=0.1$ and $K_{0}=20$. The obtained results are quasi-identical to those without cylinder rotation (not shown). This means that the vertical asymptote $\mathrm{St}_{\text {bulk }}=0$ of the phenomenological mixing model is meaningful, even if, for $\mathrm{St}_{\text {bulk }} \ll 1$, it is difficult to infer from the model precise parameter values ensuring mixing efficiency.

\section{B. Residence time distributions}

We now turn our attention to more quantitative Lagrangian flow analysis. Insight into the mixing mechanisms in open flows results from analysis of the distribution of the residence times of tracers in the mixing zone. The residence time of a tracer in the mixing zone is the time spent by the tracer within the mixing channel [44]. For simplicity, we monitor the evolution of Lagrangian tracers along the entire flow channel. At the initial time $t=0,10000$ tracers are uniformly distributed over the inlet line. Their trajectories are determined by solving:

$$
\left\{\begin{array}{l}
\dot{\boldsymbol{x}}(t)=\boldsymbol{v}[\boldsymbol{x}(t), t], \\
\boldsymbol{x}(0)=\boldsymbol{x}_{0},
\end{array}\right.
$$

which describes the evolution of the positions $\boldsymbol{x}$ of the tracers released at $\boldsymbol{x}_{0}$, and represents the fact that the rate of change of position of each tracer is given by its velocity. Here, $\boldsymbol{v}$ is the velocity field obtained by the direct simulations.

We first consider the flow without rotation of the cylinders $\left(K_{0}=0\right)$. This is approximately a Poiseuille flow, which can be seen as the worst of the cases considered in the present study in terms of mixing efficiency, since it exhibits no transverse displacements of the tracers. A log-log plot of the residence time distribution, shown in Fig. 9, suggests 
that the statistical distribution follows approximately a power law $t^{-\gamma}$ with an exponent $\gamma$ between -2 and -2.5 . Adapting the calculation proposed by Raynal and Carrière [50] for the 3-D Poiseuille flow to the 2-D case, we obtain that the exponent is equal to -3 . Given that the cylinder protuberances in the mixer channel have the effect of slowing down the evacuation of the tracers downstream in comparison to the channel considered in the theoretical case, the value of the exponent obtained numerically is in agreement with the theoretical value. The approximate algebraic

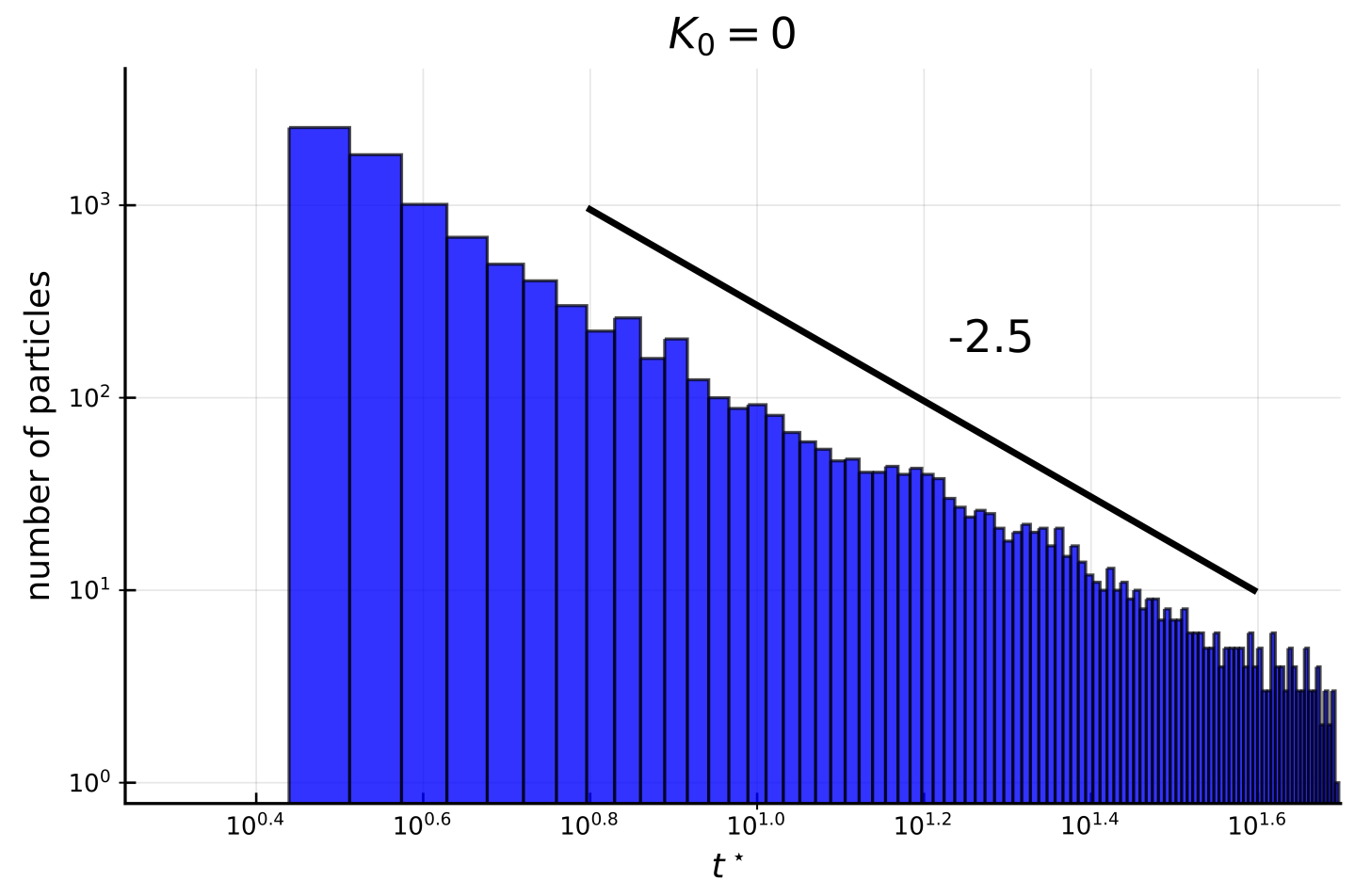

FIG. 9: Log-log plots of the residence time distribution of 10000 tracers in the mixing channel without rotation of

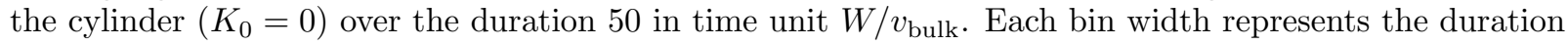
$0.5 \mathrm{~W} / v_{\text {bulk }}$.

decay rate is also evidenced in Fig. 10a by comparison with an exponential decay rate. In contrast, the cases with rotations of the cylinders shown in Figs. 10b to 10d exhibit decay rates becoming approximately exponential as the parameters $\mathrm{St}_{\mathrm{bulk}}$ and $K_{0}$ are favorable to the mixing efficiency (in agreement with the literature on chaotic advection in open flows, e.g. $[28,58])$. This exponential decay rate is observed within time intervals in which a constant fraction of tracers, $\eta$, leave the mixing zone during each period. Indeed, at the end of the cycle $n-1$ of stretching and folding operations (as described in section IIIC), the number of tracers present in the mixing zone is

$$
N=N_{0}(1-\eta)^{n-1}
$$

Using $t=(n-1) T$ and the estimate $\eta \sim v_{\text {bulk }} T / L_{\text {mixing }}$, we have

$$
N \sim N_{0} \exp \left[\frac{t}{T} \ln \left(1-\frac{v_{\text {bulk }} T}{L_{\text {mixing }}}\right)\right],
$$

and for $\zeta \mathrm{St}_{\text {bulk }}$ sufficiently large with respect to unity:

$$
N \sim N_{0} \mathrm{e}^{-t^{\star} / \zeta}
$$

where $t^{\star}=t /\left(W / v_{\text {bulk }}\right)$. Here $\zeta=5$, so that $-1 / \zeta=-0.2$, which is approximately the slope that can be observed in Figs. 10c and 10d within time intervals in which the escape rates of the tracers are approximately constant.

Notice that, as the parameters $\mathrm{St}_{\text {bulk }}$ and $K_{0}$ become favorable for a a high mixing efficiency according to the discussion of section $\mathrm{VA}$, the mean and the standard deviation of the residence times of the 10000 tracers decrease (see Table II), which can be of major practical interest, particularly in industrial applications. 


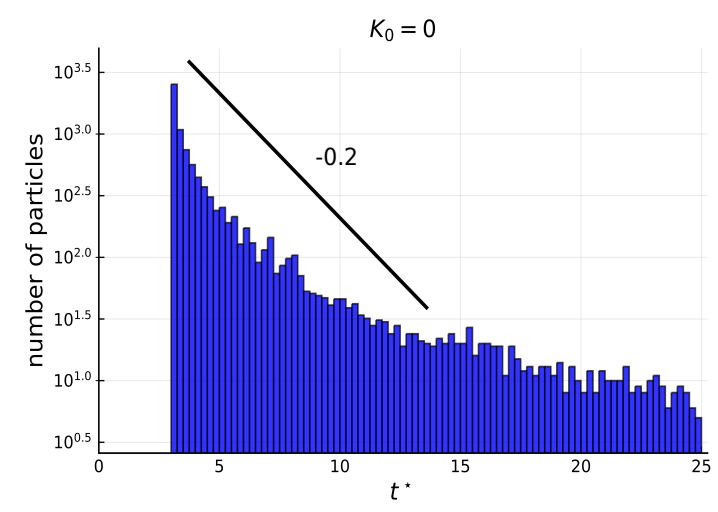

(a) $K_{0}=0$ : no cylinder rotation. 9494 tracers in 10000 have left the mixing zone at $t^{\star}=25$

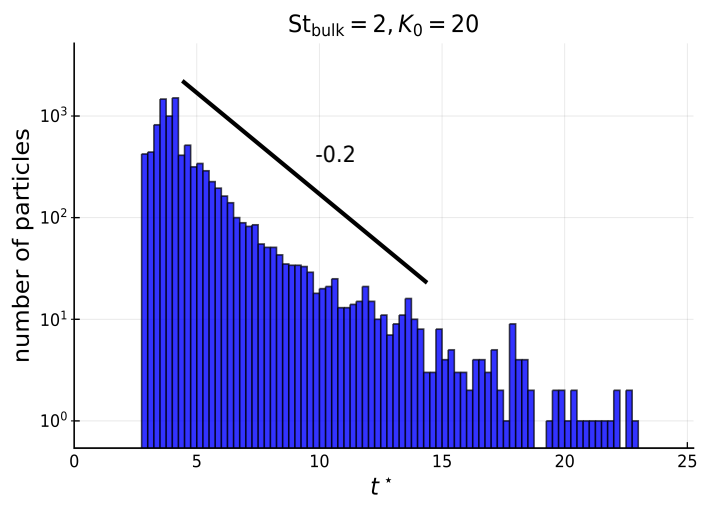

(c) $\mathrm{St}_{\text {bulk }}=2$ and $K_{0}=20.9321$ tracers in 10000 have left the mixing zone at $t^{\star}=25$.

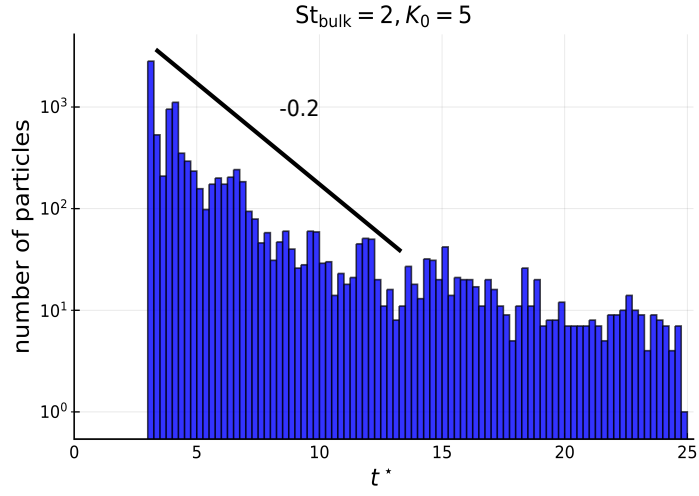

(b) $\mathrm{St}_{\text {bulk }}=2$ and $K_{0}=5.9516$ tracers in 10000 have left the mixing zone at $t^{\star}=25$

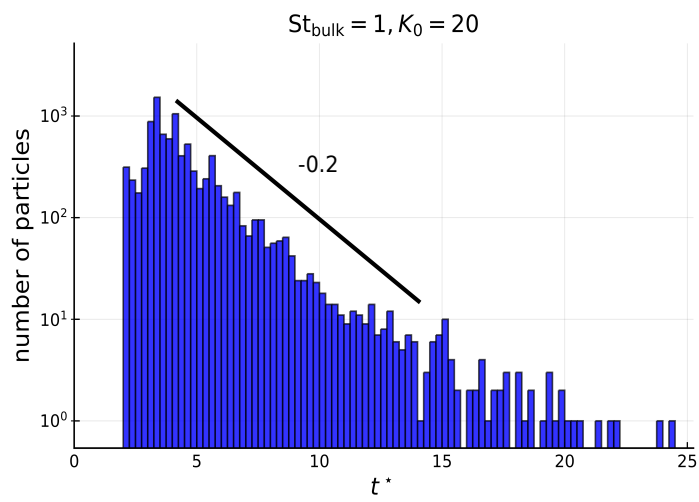

(d) $\mathrm{St}_{\text {bulk }}=1$ and $K_{0}=20.9427$ tracers in 10000 have left the mixing zone at $t^{\star}=25$.

FIG. 10: Semi-log plots of the residence time distribution of 10000 tracers in the mixing channel over the duration 25 in time unit $W / v_{\text {bulk }}$. Each bin width represents the duration $0.25 \mathrm{~W} / v_{\text {bulk }}$.

\begin{tabular}{|l|c|c|}
\hline & $\overline{\mathrm{RT}}\left(W / v_{\text {bulk }}\right)$ & $\sigma_{\mathrm{RT}}\left(W / v_{\text {bulk }}\right)$ \\
\hline$K_{0}=0$ & 5.60 & 4.00 \\
$\mathrm{St}_{\text {bulk }}=2, K_{0}=5$ & 5.48 & 3.84 \\
$\mathrm{St}_{\text {bulk }}=2, K_{0}=20$ & 4.75 & 2.15 \\
$\mathrm{St}_{\text {bulk }}=1, K_{0}=20$ & 4.54 & 2.07 \\
\hline
\end{tabular}

TABLE II: Mean $(\overline{\mathrm{RT}})$ and standard deviation $\left(\sigma_{\mathrm{RT}}\right)$ of the residence time of the tracers, among 10000 , that have left the mixing channel at $t^{\star}=25$ (in time unit $W / v_{\text {bulk }}$ ).

\section{Ageing of seeding tracers}

In Fig. 11 are shown the trajectories of 100 tracers coloured by their ages for various values of the parameters $\left(\mathrm{St}_{\text {bulk }}, K_{0}\right)$. At the initial time $t=0$, the tracers are uniformly distributed over the inlet line, at the left of each frame. Considering first the case without cylinder rotation $\left(K_{0}=0\right)$, it is shown in Fig. 11a that, to a large extent, the uniformity of the tracer distribution is preserved all along the flow channel. At the end of the simulation, the seeding tracers have left the channel, except for few tracers which are "trapped" near the walls. For the case with $\mathrm{St}_{\text {bulk }}=2$ and $K_{0}=5$ (unfavorable for mixing, as shown in section VA), it is visible in Fig. 11b that the uniformity of the tracer distribution is rapidly lost, even at the left of the cylinder (1) (see Fig. 1). Again, few tracers are trapped near the walls. The same observations can be made for the case with $\mathrm{St}_{\text {bulk }}=2$ and $K_{0}=20$ in Fig. 11c, which is in between favorable and unfavorable for mixing according to the results shown in section V A. Moreover, loops of the 
tracer trajectories begin to appear in the vicinity of the rotating cylinders.

Considering now the case with $\mathrm{St}_{\text {bulk }}=1$ and $K_{0}=20$, which is clearly favorable for mixing according to the results shown in section VA, it is apparent in Fig. 11d that the ageing distribution of the seeding tracers exhibits irregularities inside the mixing zone, contrary to what is observed in the other cases. The filamental patterns of different colours in Fig. 11d are the mark of the sensitivity to the initial conditions, and hence the signature of chaotic advection. Some filamental patterns of different colors are also visible in Fig. 11e for the case $\mathrm{St}_{\text {bulk }}=1$ and $K_{0}=40$, which is better for mixing efficiency than the case $\mathrm{St}_{\mathrm{bulk}}=1$ and $K_{0}=20$ according to Fig. 13. The relationship between mixing efficiency and the level of chaotic advection has to be further investigated, but we can already note that the case favorable for mixing is associated with chaotic advection, which is absent in the cases not clearly favorable for mixing. This supports a well-established general correlation between good mixing capacity and presence of chaotic advection (see e.g. [57]). Incidentally, the results shown in Figs. 11d and 11e also demonstrate that the proposed RAW mixer is able to exhibit chaotic mixing. 


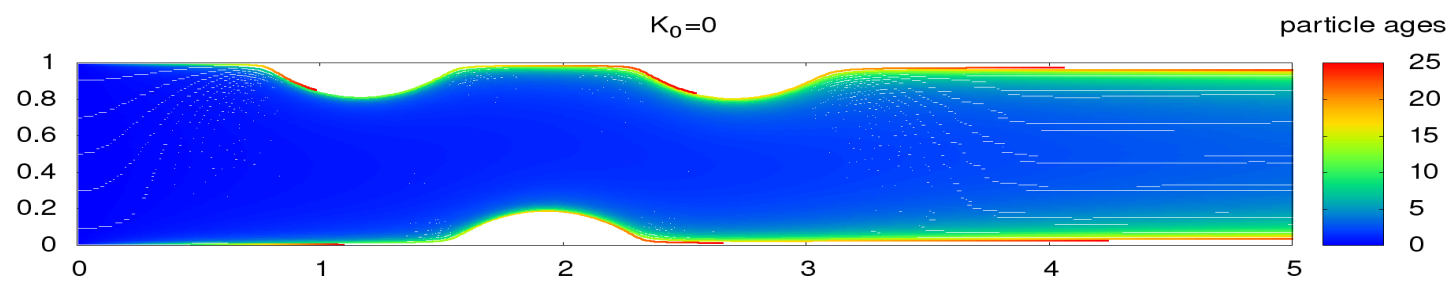

(a)

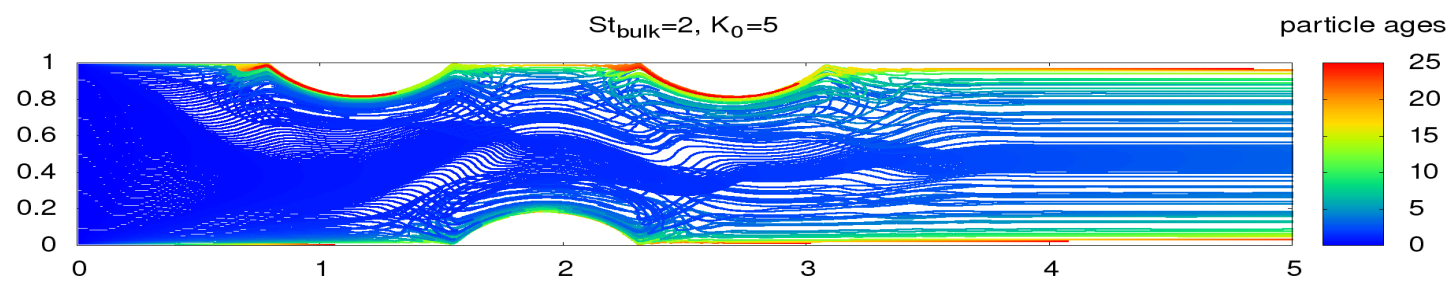

(b)

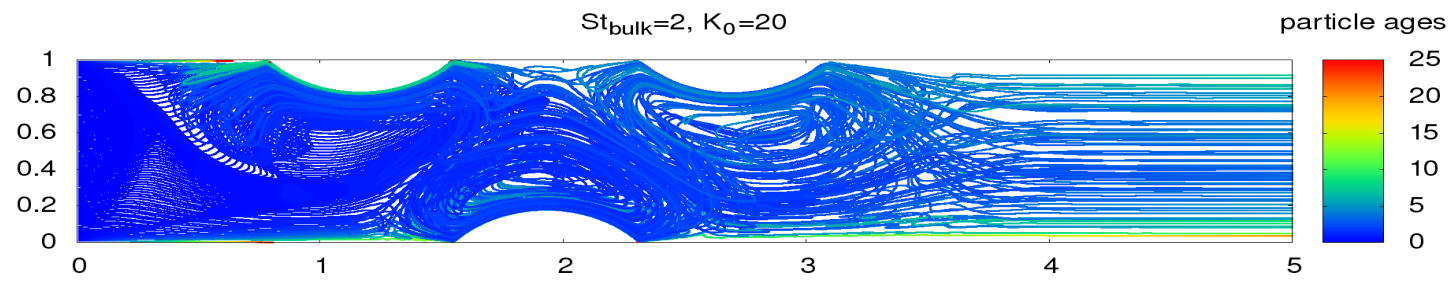

(c)

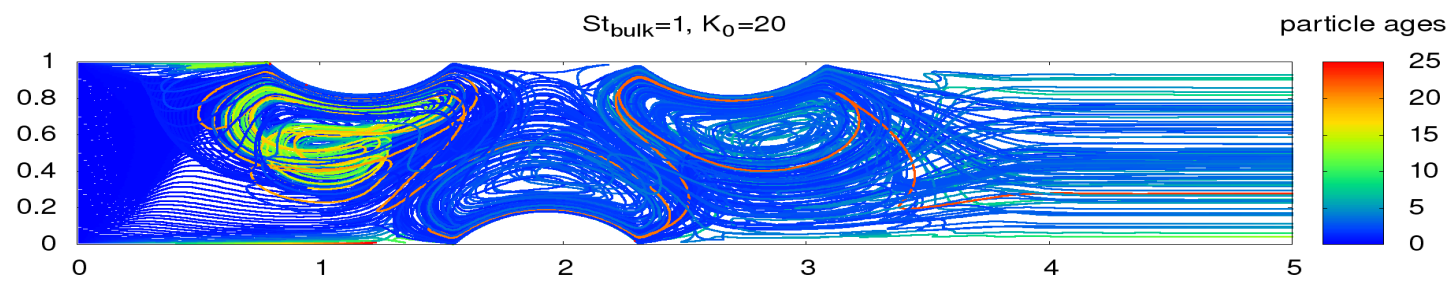

(d)

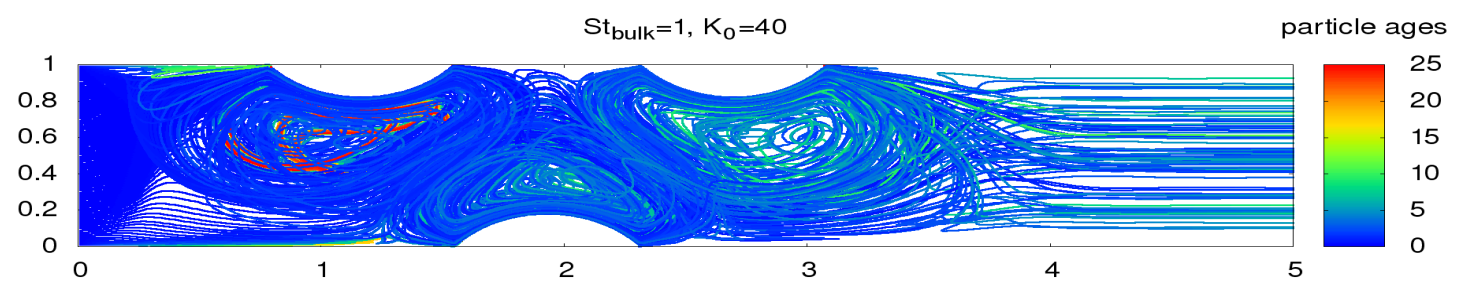

(e)

FIG. 11: Trajectories of 100 tracers in the mixing channel, colored by their ages. The duration is 25 in time unit $W / v_{\text {bulk }}$. 
The basic difference in the flow dynamics between cases clearly favorable or not for mixing is evidenced in Fig. 12 . The trajectories of 50 seeding tracers, uniformly distributed at the initial time over the lower half of the inlet line at the left of each frame, are coloured by the tracer age. It is shown that only in the case favorable for mixing $\left(\mathrm{St}_{\mathrm{bulk}}=1\right.$ and $K_{0}=20$, see Fig. 12b), large transverse displacements of the fluid tracers occur on the whole mixing zone.

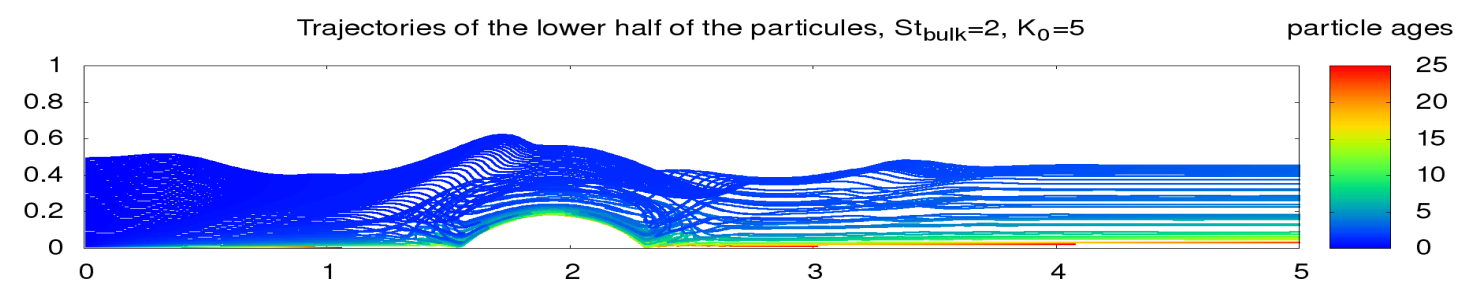

(a)

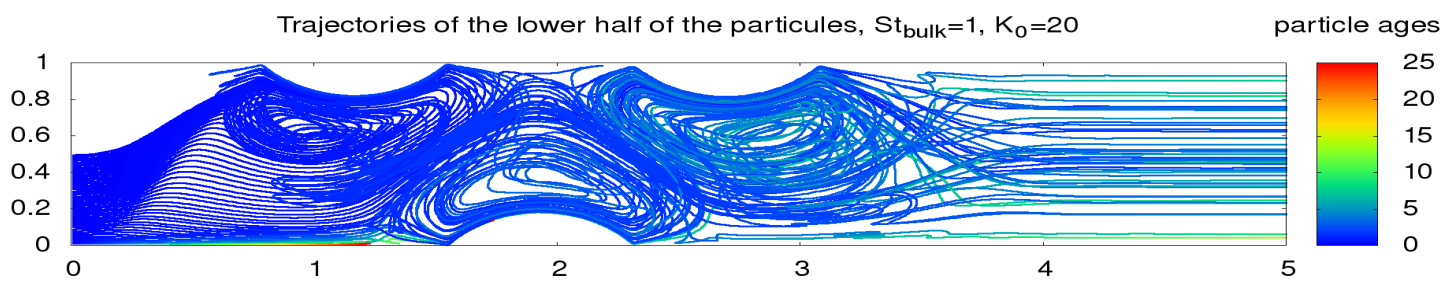

(b)

FIG. 12: Trajectories of 50 tracers in the mixing channel, colored by their ages. The tracers are injected only over the lower half of the inlet line at the left of the frames. The duration is 25 in time unit $W / v_{\text {bulk. }}$.

\section{Mixing efficiency}

In Fig. 13 are shown the contour lines of the time averaged standard deviation (27) of the concentration $c$ as a function of the bulk Strouhal number $\mathrm{St}_{\text {bulk }}$ and the ratio of velocities $K_{0}$. Are also shown the results obtained from the heuristic conditions (8) and (22) presented in section III C.

For the direct simulations, at fixed values of $K_{0}$, of the bulk Reynolds number $\mathrm{Re}_{\mathrm{bulk}}$, and of the bulk Péclet number $\mathrm{Pe}_{\text {bulk }}$, the bulk Strouhal number $\mathrm{St}_{\text {bulk }}$ dependence of the mixing efficiency represented by $\sigma$ exhibits a maximum. When the bulk Strouhal number exceeds a critical value, the mixing efficiency decreases for large Strouhal number, and increases with the Strouhal number below the critical value. Thus, the direct simulations conform with theoretical suggestions by Rom-Kedar and Poje [54] for flows with hyperbolic saddles, and with numerical and experimental observations by Horner et al. [32] for flows with parabolic-type saddles.

The variations of the mixing level as a function of the control parameters of the mixer obtained from the direct simulations are in good agreement with the results obtained from the heuristic conditions. Even if the mixing level itself is known only approximately, the heuristic model thus provides useful information on the control parameters to be chosen for reaching mixing efficiency without performing a large number of costly direct simulations. We believe that this is remarkable in itself, as our heuristic model was minimalistic and did not account for all the details of the the complex flow revealed by the numerical simulations.

\section{CONCLUSION}

An active mixing device for open flows was presented, along with some numerical results on the mixing of a passive scalar concentration in a Newtonian fluid at low Reynolds number and high Péclet number in the two-dimensional case. The flow modulation protocol is imposed by three rotating circular arc-walls in a straight channel. The selected scenario of a smooth alternation of the direction rotations of the cylinders allows for the stretching of concentration areas and the folding of concentration filaments, which are ordered processes known to be suitable for good mixing of a passive scalar concentration. Two simple heuristic conditions based on phenomenological arguments were presented, 


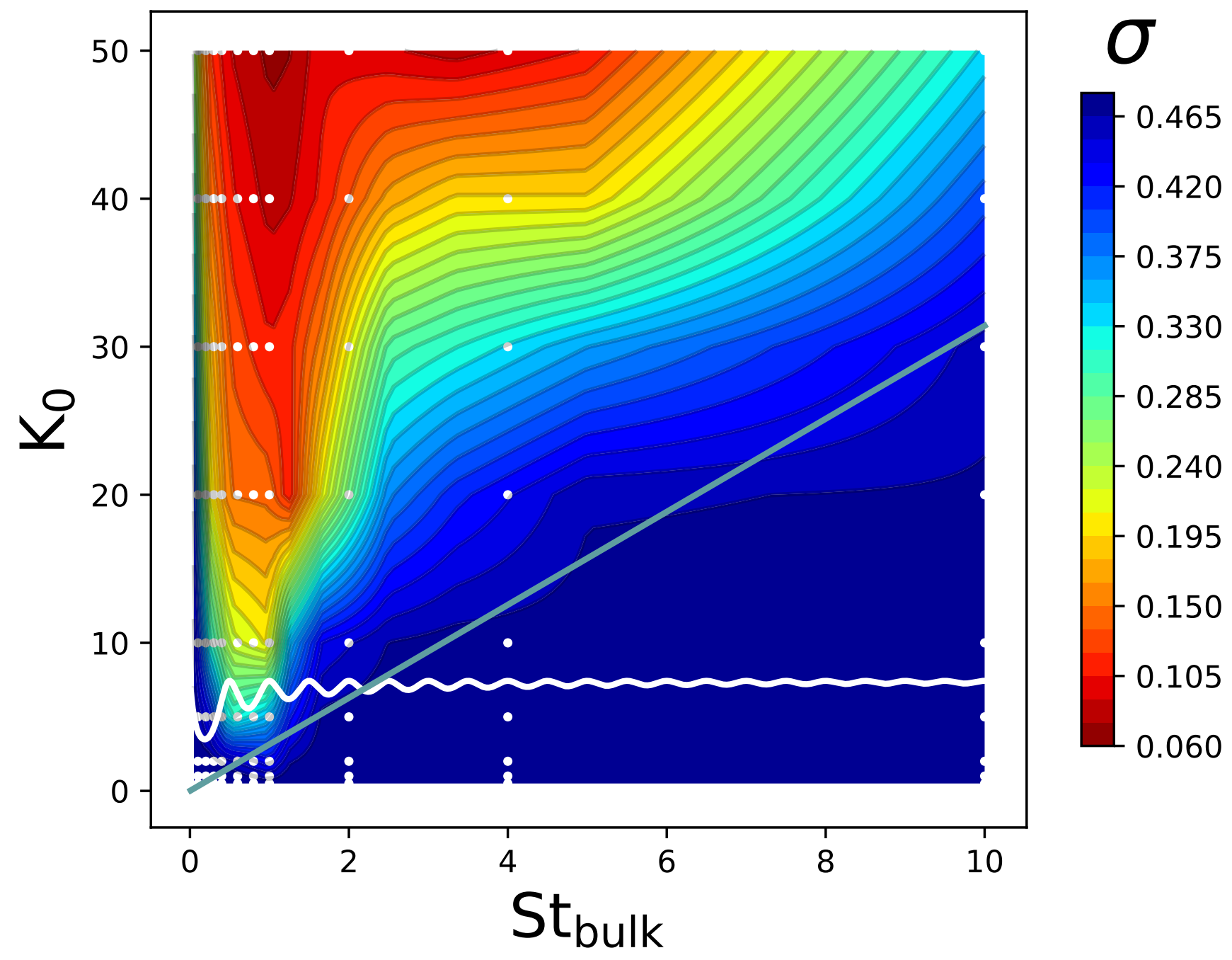

FIG. 13: Standard deviation of the concentration $c$ as a function of $\mathrm{St}_{\text {bulk }}$ (see Eq. (6)) and $K_{0}$ (see Eq.(8)), with $\zeta=2$ and $P e_{\text {bulk }}=10^{6}$. Each of the 90 circles $(\mathrm{o})$ corresponds to the result of a direct simulation. Linear interpolation is applied subsequently between the results. The black and white lines are the lines shown in Fig. 3, based on the folding condition (8) and the condition (22) for mixing.

one for folding and the other for mixing with scalar diffusion embedded in the model. These involve two control parameters: the Strouhal number based on the bulk flow velocity, and the strength of the cross flow relative to the transport flow. The heuristic conditions lead to values of these parameters that are in good agreement for mixing efficiency with those obtained from the direct simulations. It was thus shown that the conditions could provide useful informations on the control parameters to be selected for mixing efficiency, for example as a first attempt before mixing optimization. In the cases of good mixing efficiency, chaotic advection was detected in the flow both through the study of the tracer trajectories and the residence time distribution profiles. The beneficial role of the elliptic regions of the flow, generated by the rotating walls, for stretching and folding the concentration areas, was evidenced. The active inline RAW mixer is thus promising for producing efficiently chaotic mixing, in particular by the adaptability of the mixing protocols that can be chosen.

\section{ACKNOWLEDGMENTS}

The authors acknowledge the financial support from the french research program ANR-16-CE06-0003 through the NaiMYS project (Novel active inline Mixer for Yield Stress fluids). The computations were carried out on 
the Avakas cluster of the Mésocentre de Calcul Intensif Aquitain (MCIA) and the Occigen supercomputer of the Centre Informatique National de l'Enseignement Supérieur (CINES). The authors are grateful to A. Achhoud for his participation to the preliminary numerical tests as a part of his MsC studies at University of Pau and Pays de l'Adour.

[1] O. M. Aamo, M. Krstić, T. R. Bewley. Control of mixing by boundary feedback in 2D channel flow. Automatica, 39:1597$1606,2003$.

[2] S. Ali, C. Habchi, S. Menanteau, T. Lemenand, and J.-L. Harion. Heat transfer and mixing enhancement by free elastic flaps oscillation. Int. J. Heat Mass Transf., 85:250-264, 2015.

[3] C. H. Amon, A. M. Guzmán, and B. Morel. Lagrangian chaos, Eulerian chaos, and mixing enhancement in converging-diverging channel flows. Phys. Fluids, 8:1192, 1996.

[4] H. Aref, J. R. Blake, M. Budišić, S. S. S. Cardoso, J. H. E. Cartwright, H. J. H. Clercx, K. El Omari, U. Feudel, R. Golestanian, E. Gouillart, G. F. van Heijst, T. S. Krasnopolskaya, Y. Le Guer, R. S. MacKay, V. V. Meleshko, G. Metcalfe, I. Mezić, A. P. S. de Moura, O. Piro, M. F. M. Speetjens, R. Sturman, J.-L. Thiffeault, and I. Tuval. Frontiers of chaotic advection. Rev. Mod. Phys., 89:025007, 2017.

[5] L. Bammou, K. El Omari, S. Blancher, Y. Le Guer, B. Benhamou, and T. Mediouni. A numerical study of the longitudinal thermoconvective rolls in a mixed convection flow in a horizontal channel with a free surface. Int. J. Heat Fluid Flow, 42:265-277, 2013.

[6] J. J. B. Biemond, A. P. S. De Moura, G. Károlyi, C. Grebogi, H. Nijmeijer. Onset of chaotic advection in open flows. Phys. Rev. E, 78:016317, 2008.

[7] C. Boesinger, Y. Le Guer, and M. Mory. Experimental study of reactive chaotic flows in tubular reactors. AIChE J., $51: 2122-2132,2005$.

[8] F. Bottausci, I. Mezić, C. D. Meinhart, and C. Cardonne. Mixing in the shear superposition micromixer: three-dimensional analysis. Philos. Tr. R. Soc. A, 362:1001-1018, 2004.

[9] T. Burghelea, E. Segre, I. Bar-Joseph, A. Groisman, and V. Steinberg. Chaotic flow and efficient mixing in a microchannel with a polymer solution. Phys. Rev. E, 69:8, 2004.

[10] T. Burghelea, E. Segre, and V. Steinberg. Mixing by polymers: experimental test and decay regime of mixing. Phys. Rev. Lett., 92:164501, 2004.

[11] G. Cai, L. Xue, H. Zhang, and J. Lin. A review on micromixers. Micromachines, 8:274-300, 2017.

[12] C. Castelain, A. Mokrani, Y. Le Guer, and H. Peerhossaini. Experimental study of chaotic advection regime in a twisted duct flow. Eur. J. Mech. B - Fluids, 20:205-232, 2001.

[13] C.-C. Chang and R.-J. Yang. Electrokinetic mixing in microfluidic systems. Microfluid. Nanofluid., 3:501-525, 2007.

[14] D. J. Collins, Z. Ma, J. Han, and Y. Ai. Continuous micro-vortex-based nanoparticle manipulation via focused surface acoustic waves. Lab Chip, 17:91-103, 2017.

[15] D. D'Alessandro, M. Dahleh, and I. Mezić. Control of mixing in fluid flow: a maximum entropy approach. IEEE Trans. Automat. Contr., 44:1852-1863, 1999.

[16] P. V. Danckwerts. The definition and measurement of some characteristics of mixtures. Appl. Sci. Res., 3:279-296, 1952.

[17] A. de Moura, U. Feudel, and E. Gouillart. Mixing and chaos in open flows. Adv. Appl. Mech., 45:1-50, 2012.

[18] J. D. den Toonder, F. Bos, D. Broer, L. Filippini, M. Gillies, J. de Goede, T. Mol, M. Reijme, W. Talen, H. Wilderbeek, V. Khatavkar, and P. D. Anderson. Artificial cilia for active micro-fluidic mixing. Lab Chip, 8:533-541, 2008.

[19] C. Eckart. An analysis of the stirring and mixing processes in incompressible fluids. J. Mar. Res., 7:265-275, 1948.

[20] K. El Omari, A. Achhoud and Y. Le Guer. Chaotic mixing in a plane channel with rotating arc walls. In 62nd Annual Meeting of the APS Division of Fluid Dynamics (American Physical Society, New York, 2009), Vol. 54.

[21] K. El Omari and Y. Le Guer. Alternate rotating walls for thermal chaotic mixing. Int. J. Heat Mass Transf., 53:123-134, 2010.

[22] K. El Omari and Y. Le Guer. Thermal chaotic mixing of power law fluids in a mixer with alternately-rotating walls. J. Non-Newtonian Fluid Mech., 165:641-651, 2010.

[23] M. D. Finn, S. M. Cox, and H. M. Byrne. Mixing measures for a two-dimensional chaotic Stokes flow. J. Eng. Math., 48:129-155, 2004.

[24] D. P. G. Foures, C. P. Caulfield, and P. J. Schmid. Optimal mixing in two-dimensional plane Poiseuille flow at finite Péclet number. J. Fluid Mech., 748:241-277, 2014.

[25] S. W. Gepner and M. Floryan. Flow dynamics and enhanced mixing in a converging-diverging channel. J. Fluid Mech., 807:167-204, 2016.

[26] A. Ghanem, C. Habchi, T. Lemenand, D. Della Valle, and H. Peerhossaini. Mixing performances of swirl flow and corrugated channel reactors. Chem. Eng. Res. Des., 92:2213-2222, 2014.

[27] S. Gopalakrishnan and A. Thess. Electromagnetically induced chaotic mixing in a pipe mixer. Chem. Engng. Sci., 65:62826291, 2010.

[28] E. Gouillart, O. Dauchot, J.-L. Thiffeault, and S. Roux. Open-flow mixing: experimental evidence for strange eigenmodes. Phys. Fluids, 21:023603, 2009.

[29] A. Groisman and V. Steinberg. Efficient mixing at low Reynolds numbers using polymer additives. Nature, 410:905-908, 2001. 
[30] G. Haller. Lagrangian coherent structures. Annu. Rev. Fluid Mech., 47:137-161, 2015.

[31] V. Hessel, H. Löwe, and F. Schönfeld. Micromixers: a review on passive and active mixing principles. Chem. Engng Sci., 60:2479-2501, 2005.

[32] M. Horner, G. Metcalfe, S. Wiggins, J. M. Ottino. Transport enhancement mechanisms in open cavities. J. Fluid Mech., 452:199-229, 2002.

[33] P.-H. Huang, Y. Xie, D. Ahmed, J. Rufo, N. Nama, Y. Chen, C. Y. Chan, and T. J. Huang. An acoustofluidic micromixer based on oscillating sharp-edges. Lab Chip, 13:3847-3852, 2013.

[34] S. W. Jones, O. M. Thomas, and H. Aref. Chaotic advection by laminar flow in a twisted pipe. J. Fluid Mech., 209:335-357, 1989.

[35] D. V. Khakhar, J. G. Franjione, and J. M. Ottino. A case study of chaotic mixing in deterministic flows: the partitioned-pipe mixer. Chem. Eng. Sci., 42:2909-2926, 1987.

[36] V. V. Khatavkar, P. D. Anderson, J. M. J. den Toonder, and H. E. H. Meijer. Active micromixer based on artificial cilia. Phys. Fluids, 19:083605, 2007.

[37] J. B. Knight, A. Vishwanath, J. P. Brody, and R. H. Austin. Hydrodynamic focusing on a silicon chip: mixing nanoliters in microseconds. Phys. Rev. Lett., 80:3863, 1998.

[38] R. A. Lambert and R. H. Rangel. The role of elastic flap deformation on fluid mixing in a microchannel. Phys. Fluids, 22:052003, 2010.

[39] Y. Le Guer and K. El Omari. Chaotic advection for thermal mixing. Adv. Appl. Mech., 45:189-237, 2012.

[40] H.-Y. Lee and J. Voldman. Optimizing micromixer design for enhancing dielectrophoretic microconcentrator performance. Anal. Chem., 79:1833-1839, 2007.

[41] Z. Lin, J.-L. Thiffeault, and C. R. Doering. Optimal stirring strategies for passive scalar mixing. J. Fluid Mech., 675:465476, 2011.

[42] G. Mathew, I. Mezić, and L. Petzold. A multiscale measure for mixing. Phys. D, 211:23-46, 2005.

[43] P. Meunier and E. Villermaux. How vortices mix. J. Fluid Mech., 476:213-222, 2003.

[44] E. B. Nauman. Residence time theory. Ind. Eng. Chem. Res., 47:3752-3766, 2008.

[45] T. J. Ober, D. Foresti, and J. A. Lewis. Active mixing of complex fluids at the microscale. P. Nat. Acad. Sci. USA, 112:12293-12298, 2015.

[46] M. H. Oddy, J. G. Santiago, and J. C. Mikkelsen. Electrokinetic instability micromixing. Anal. Chem., 73:5822-5832, 2001.

[47] F. Okkels and P. Tabeling. Spatiotemporal resonances in mixing of open viscous fluids. Phys. Rev. Lett., 92:038301, 2004.

[48] Z. Pouransari, M.F. M. Speetjens, and H. J. H. Clercx. Formation of coherent structures by fluid inertia in three-dimensional laminar flows. J. Fluid Mech., 654:5-34, 2010.

[49] B. Rallabandi, C. Wang, and S. Hilgenfeldt. Analysis of optimal mixing in open-flow mixers with time-modulated vortex arrays. Phys. Rev. Fluids, 2:064501, 2017.

[50] F. Raynal and P. Carrière. The distribution of "time of flight" in three dimensional stationary chaotic advection. Phys. Fluids, 27:043601, 2015.

[51] B. Ravu, G. Metcalfe, M. Rudman, D. R. Lester, and D. V. Khakhar. Global organization of three-dimensional, volumepreserving flows: Constraints, degenerate points, and Lagrangian structure. Chaos, 30:033124, 2020.

[52] P. B. Rhines and W. R. Young. How rapidly is a passive scalar mixed within closed streamlines? J. Fluid Mech., 133:133$145,1983$.

[53] A. J. S. Rodrigo, J. P. B. Mota, A. Lefèvre, and E. Saatdjian. On the optimization of mixing protocol in a certain class of three-dimensional Stokes flows. Phys. Fluids, 15:1505-1516, 2003.

[54] V. Rom-Kedar and A. C. Poje. Universal properties of chaotic transport in the presence of diffusion. Phys. Fluids, 11:20442057, 1999.

[55] H. A. Stone, A. D. Stroock, and A. Ajdari. Engineering flows in small devices: microfluidics toward a Lab-on-a-Chip. Annu. Rev. Fluid Mech., 36:381-411, 2004.

[56] A. D. Stroock, S. K. W. Dertinger, A. Ajdari, I. Mezić, H. A. Stone, and G. M. Whitesides. Chaotic mixer for microchannels. Science, 295:647-651, 2002.

[57] P. Sundararajan and A. D. Stroock. Transport phenomena in chaotic laminar flows. Annu. Rev. Chem. Biomol. Eng., 3:473-496, 2012.

[58] T. Tél, G. Károlyi, Á. Péntek, I. Scheuring, Z. Toroczkai, C. Grebogi, and J. Kadtke. Chaotic advection, diffusion, and reactions in open flows. Chaos, 10:89, 2000.

[59] J.-L. Thiffeault. Using multiscale norms to quantify mixing and transport. Nonlinearity, 25:R1, 2012.

[60] E. Villermaux. Mixing versus stirring. Annu. Rev. Fluid Mech.,51:245-273, 2019. 